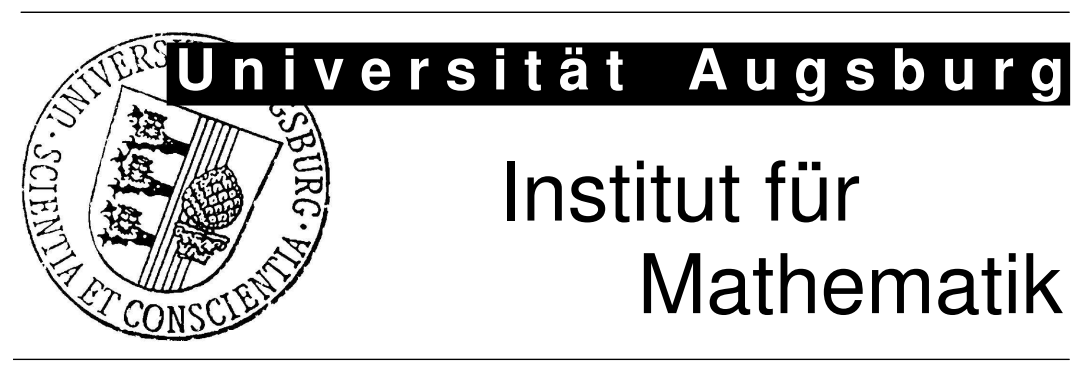

Lothar Heinrich

Gaussian Limits of Empirical Multiparameter K-Functions of Homogeneous Poisson Processes and Tests for Complete Spatial Randomness 


\section{Impressum:}

\section{Herausgeber:}

Institut für Mathematik

Universität Augsburg

86135 Augsburg

http://www . math. uni-augsburg.de/de/forschung/preprints.html

\section{ViSdP:}

Lothar Heinrich

Institut für Mathematik

Universität Augsburg

86135 Augsburg

Preprint: Sämtliche Rechte verbleiben den Autoren (C) 2014 


\title{
GAUSSIAN LIMITS OF EMPIRICAL MULTIPARAMETER K-FUNCTIONS OF HOMOGENEOUS POISSON PROCESSES AND TESTS FOR COMPLETE SPATIAL RANDOMNESS
}

\author{
Lothar Heinrich
}

\author{
University of Augsburg, Institute of Mathematics, 86135 Augsburg, Germany \\ ( e-mail: heinrich@math.uni-augsburg.de)
}

\begin{abstract}
We prove two functional limit theorems for empirical multiparameter second moment functions (generalizing Ripley's $K$-function) obtained from a homogeneous Poisson point field observed in an unboundedly expanding convex sampling window $W_{n}$ in $\mathbb{R}^{d}$. The cases of known and unknown (estimated) intensity lead to distinct Gaussian limits and require quite different proofs. Further we determine the limit distributions of the maximal deviation and the integrated squared distance between empirical and true multiparameter second moment function. These results give rise to construct goodness-of-fit tests for checking the hypothesis that a given point pattern is completely spatially random (CSR), i.e. a realization of a homogeneous Poisson process.
\end{abstract}

Keywords: Point process, reduced second moment measure, set-indexed Gaussian process, multiparameter Gaussian process, Wiener sheet, m-dependence, U-statistic, weak convergence, Skorokhodspace of multiparameter càdlàg-functions, goodness-of-fit tests

MSC 2010: Primary 60 F 17, 60 G 55; Secondary 60 F 05, 60 G 60

\section{Introduction and Main Results}

Statistical second-order analysis of spatially homogeneous point fields observed in a (large) bounded region (called sampling window) $W_{n}$ of the $d$-dimensional Euclidean space $\mathbb{R}^{d}$ is based on the (asymptotic) properties of the empirical intensity $\hat{\lambda}_{n}=N\left(W_{n}\right) /\left|W_{n}\right|$ and the empirical process

$$
X_{n}(B)=\sum_{i, j \geq 1}^{\neq} \mathbf{1}_{W_{n}}\left(X_{i}\right) \mathbf{1}_{W_{n}}\left(X_{j}\right) \mathbf{1}_{B}\left(X_{j}-X_{i}\right) \quad \text { for } \quad B \in \mathcal{B}^{d} \cap C_{\varrho},
$$

where $N=\sum_{i \geq 1} \delta_{X_{i}}$ denotes a simple stationary point process on $\mathbb{R}^{d}$ (defined as locally finite random counting measure without multiple atoms on the Borel sets $\mathcal{B}^{d}$ of $\mathbb{R}^{d}$ ) and $C_{\varrho}$ stands for the cube $[-\varrho, \varrho]^{d}$ centred at the origin $\mathbf{o}$ with a freely selectable parameter $\varrho>0$. In (1.1) and throughout the paper, the sum $\sum^{\neq}$runs over pairwise distinct indices, $\delta_{x}$ and $\mathbf{1}_{B}$ are connected by $\delta_{x}(B)=\mathbf{1}_{B}(x)=1$ for $x \in B$ and $=0$ for $x \notin B$ and $|B|$ denotes the Lebesgue 
measure of $B \in \mathcal{B}_{b}^{d}$ (= family of bounded members of $\mathcal{B}^{d}$ ). Further, all random variables and processes are defined over a common probability space $[\Omega, \mathcal{F}, \mathbf{P}]$ (which always exists). To study the asymptotic behaviour of $(1.1)$ we assume that the sequence $\left(W_{n}\right)$ forms a convex averaging sequence (briefly CAS), i.e. $\left(W_{n}\right)$ consists of increasing, convex and compact sets in $\mathbb{R}^{d}$ such that $r\left(W_{n}\right):=\sup \left\{r>0: B(x, r) \subseteq W_{n}\right.$ for some $\left.x \in W_{n}\right\} \longrightarrow \infty$ as $n \rightarrow \infty$ (or equivalently $\left|\partial W_{n}\right|_{d-1} /\left|W_{n}\right| \longrightarrow 0$ as $\left.n \rightarrow \infty\right)$, where $B(x, r)$ denotes the closed Euclidean ball with center $x \in \mathbb{R}^{d}$ and radius $r \geq 0$, and $\left|\partial W_{n}\right|_{d-1}$ indicates the surface content of $W_{n}$.

By applying some of results from convex geometry to the CAS $\left(W_{n}\right)$ it can be shown that

$$
\frac{1}{r\left(W_{n}\right)} \leq \frac{\left|\partial W_{n}\right|_{d-1}}{\left|W_{n}\right|} \leq \frac{d}{r\left(W_{n}\right)} \quad \text { and } \quad \frac{\varrho}{r\left(W_{n}\right)} \leq \frac{\left|W_{n} \oplus B(\mathbf{o}, \varrho)\right|-\left|W_{n}\right|}{\left|W_{n}\right|} \leq \frac{\left(2^{d}-1\right) \varrho}{r\left(W_{n}\right)}
$$

for any $\varrho \geq 0$, see [17], p.54, and [19], Appendix .

The properties of the CAS $\left(W_{n}\right)$ are essential in proving spatial limit theorems (although in some cases e.g. the convexity of $W_{n}$ can be slightly relaxed). E.g., for a stationary ergodic point process $N=\sum_{i \geq 1} \delta_{X_{i}}$ with intensity $\lambda=\mathbf{E} N\left([0,1]^{d}\right)$ satisfying $\mathbf{E} N^{2}\left([0,1]^{d}\right)<\infty$, the spatial ergodic theorem of Nguyen-Zessin, see [10] or [26], yields the $\mathbf{P}$-a.s. limits $\widehat{\lambda}_{n} \underset{n \rightarrow \infty}{\mathbf{P}-\text { a.s. }} \lambda \lambda$ and

$$
\frac{X_{n}(B)}{\left|W_{n}\right|} \underset{n \rightarrow \infty}{\stackrel{\mathbf{P}-a . s .}{\longrightarrow}} \lambda \mathbf{E}\left(\left(N-\delta_{\mathbf{o}}\right)(B) \mid N(\{\mathbf{o}\})>0\right)=: \lambda^{2} \mathcal{K}(B) \quad \text { for any } \quad B \in \mathcal{B}_{b}^{d},
$$

where the expectation of the number $\left(N-\delta_{\mathbf{o}}\right)(\cdot)$ conditional on the null event $\{N(\{o\})>$ $0\}$, abbreviated by $\lambda \mathcal{K}(\cdot)$ in what follows, coincides with the reduced second-order moment measure of $N$, see [9] (Chapt. 8), and can be rigorously defined as first-order moment measure w.r.t. the reduced Palm distribution of $N$, see [10] (Chapt. 13) for details. It turns out that the locally finite $K$-measure $\mathcal{K}(\cdot)$ is o-symmetric, i.e. $\mathcal{K}(B)=\mathcal{K}(-B)$ for $B \in \mathcal{B}_{b}^{d}$. On the other hand, to the best of the author's knowledge a complete characterization of the $K$-measure is so far still unknown. Another issue is to find suitable parametric subfamilies of $\mathcal{B}_{b}^{d}$ on which the $K$-measure is uniquely determined or at least described sufficiently well by the corresponding functions of parameters. For example, Ripley's (one-parameter) $K$ function $K(r):=\mathcal{K}(B(\mathbf{o}, r))$ for $r \geq 0$, see [37], defines uniquely the $K$-measure when the associated stationary point process $N$ is additionally isotropic, see e.g. [23], Chapt. 4.3. In the latter reference and in numerous papers, e.g. [40], [6], [12], [41], [22] , one can find various refined and tricky, partially sophisticated techniques to estimate $K(r)$ (and the related $L$ function $L(r)=(K(r))^{1 / d}$ and pair-correlation function $\left.g(r)=r K^{\prime}(r) / d|B(\mathbf{o}, r)|\right)$ which are particularly useful when the point pattern and $W_{n}$ is comparatively small. If the isotropy of $N$ is missing, then Ripley's $K$-function should be replaced by the $K$-measure on reacher parametrized families sets, e.g., increasing (one-parametric) families $\left\{r B_{s}: r \geq 0\right\}$, where $B_{s}$ is any fixed o-symmetric, convex, compact set in $\mathbb{R}^{d}$ containing $\mathbf{o}$ as inner point, which allows to measure the distance of points by the norm $n(x):=\inf \left\{\tau>0: x \notin \tau B_{s}\right\}$, see [19]. 
To exhaust as much information from the point pattern as possible we suggest to consider the $K$-measure on the following families of rectangular sets indexed by the multiparameter $\mathbf{r}=\left(r_{1}, \ldots, r_{d}\right)$ :

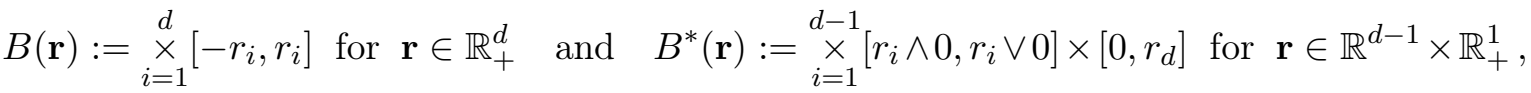
where $a \vee(\wedge) b:=\max (\min )\{a, b\}$. By standard measure-theoretic approximation arguments we can prove

Proposition 1. The function $\mathbb{R}^{d-1} \times \mathbb{R}_{+}^{1} \ni \mathbf{r} \mapsto K^{*}(\mathbf{r}):=\mathcal{K}\left(B^{*}(\mathbf{r})\right)$ determines the $K$ measure uniquely.

In the following, for reasons of symmetry and to facilitate statistical procedures we prefer to establish the multiparameter $K$-function $\mathbb{R}_{+}^{d} \ni \mathbf{r} \mapsto K(\mathbf{r}):=\mathcal{K}(B(\mathbf{r}))$ although it is open whether Proposition 1 holds for $K(\mathbf{r})$. It should be mentioned that the limit (1.3) holds even uniformly w.r.t. the class of convex polyhedra contained in a convex compact set, say $C_{\varrho}$ for some $\varrho>0$, see [26], p. 371. In case of balls $\left\{x \in \mathbb{R}^{d}: n(x) \leq r\right\}$ and hyper-rectangles $B(\mathbf{r})$ or $B^{*}(\mathbf{r})$ contained in $C_{\varrho}$ this uniform $\mathbf{P}$-a.s. convergence is easily obtained from (1.3) in analogy to the proof of the (multidimensional) Glivenko-Cantelli theorem, see [26], p. 136.

Next, we introduce two further (unbiased) set-indexed estimators for $\lambda^{2} \mathcal{K}(B)$ by modifying the estimator $\left(\widehat{\lambda^{2} \mathcal{K}}\right)_{n, 3}(B):=X_{n}(B) /\left|W_{n}\right|$ on the 1.h.s. of $(1.3)$ :

$$
\begin{aligned}
\left(\widehat{\lambda^{2} \mathcal{K}}\right)_{n, 1}(B) & \left.:=\frac{1}{\left|W_{n}\right|} \sum_{i \geq 1} \mathbf{1}_{W_{n}}\left(X_{i}\right)\left(N-\delta_{X_{i}}\right)\left(B+X_{i}\right)\right) \\
\left(\widehat{\lambda^{2} \mathcal{K}}\right)_{n, 2}(B) & :=\sum_{i, j \geq 1}^{\neq} \frac{\mathbf{1}_{W_{n}}\left(X_{i}\right) \mathbf{1}_{W_{n}}\left(X_{j}\right) 1_{B}\left(X_{j}-X_{i}\right)}{\left|\left(W_{n}-X_{i}\right) \cap\left(W_{n}-X_{j}\right)\right|}
\end{aligned}
$$

By setting $\left(\widehat{\lambda^{2} K}\right)_{n, i}(\mathbf{r}):=\left(\widehat{\lambda^{2} \mathcal{K}}\right)_{n, i}(B(\mathbf{r}))$ for $i=1,2,3$ we obtain three empirical multiparameter $K$-functions estimating $\lambda^{2} K(r)$ for $\mathbf{r} \in \mathbb{R}_{+}^{d}$. An application of the refined Campbell theorem (Campbell-Mecke formula), see [10], pp. 286-288, or of the Propositions 6.1 and 8.1 in [9] provide the following means of the above estimators:

$$
\mathbf{E}\left(\widehat{\lambda^{2} \mathcal{K}}\right)_{n, 1}(B)=\mathbf{E}\left(\widehat{\lambda^{2} \mathcal{K}}\right)_{n, 2}(B)=\lambda^{2} \mathcal{K}(B) \text { and } \mathbf{E}\left(\widehat{\lambda^{2} \mathcal{K}}\right)_{n, 3}(B)=\lambda^{2} \int_{B} R_{n}(x) \mathcal{K}(\mathrm{d} x),
$$

where $R_{n}(x):=\left|W_{n} \cap\left(W_{n}-x\right)\right| /\left|W_{n}\right|$.

In other words, the estimators (1.4) and (1.5) are unbiased, whereas the asymptotic unbiasedness of $\left(\widehat{\lambda^{2} \mathcal{K}}\right)_{n, 3}(B)$ follows by use of the first inequality of (1.2), see also [17]. Summing up the above relations and arguments combined with the properties of the CAS $\left(W_{n}\right)$, we can state the following Glivenko-Cantelli type theorem for empirical multiparameter $K$-functions: Under the assumptions of the ergodic theorem (1.3), 


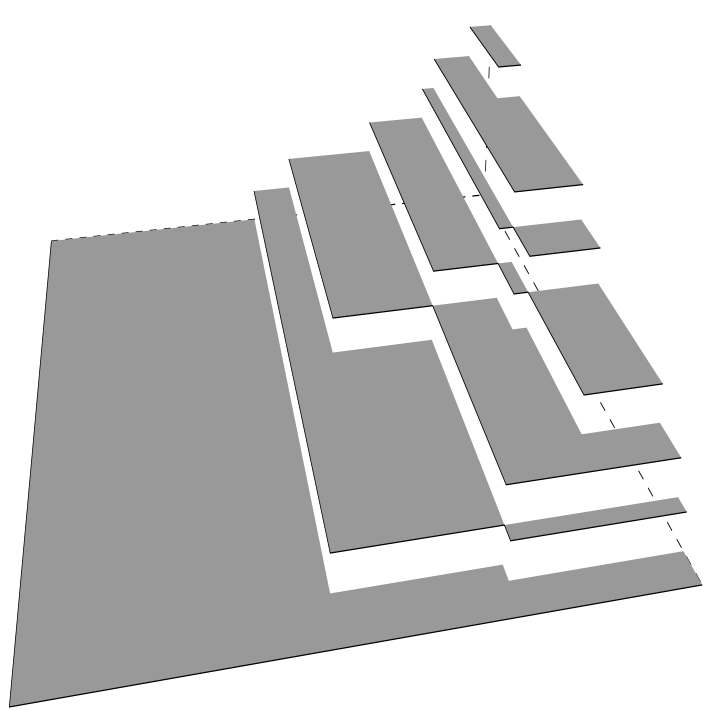

Fig.1: Plot of the empirical K-function $\left.\widehat{\lambda^{2} K}\right)_{n, 3}\left(r_{1}, r_{2}\right)$

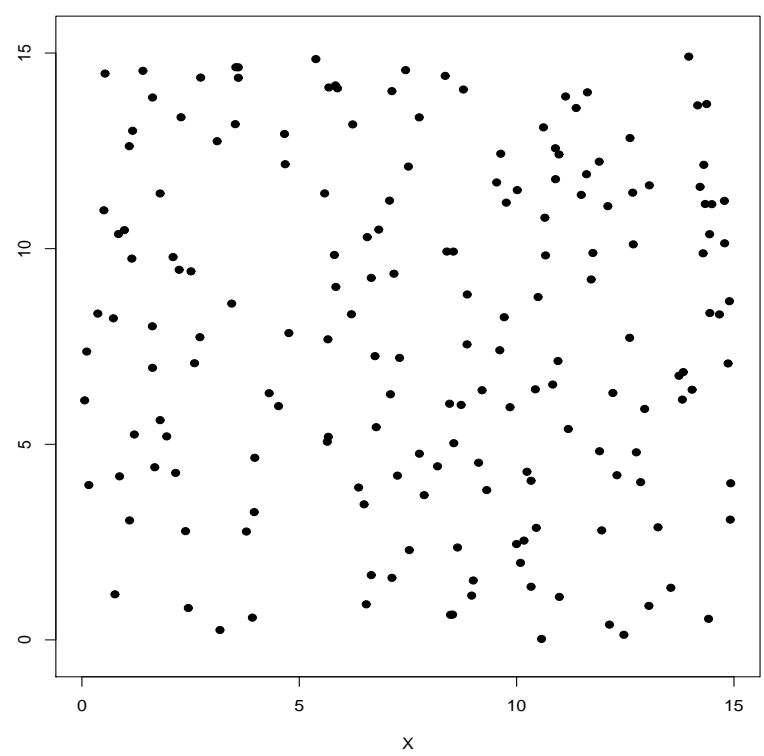

Fig.2: Simulated Poisson process $N_{0.8}$ in $[0,15]^{2}$

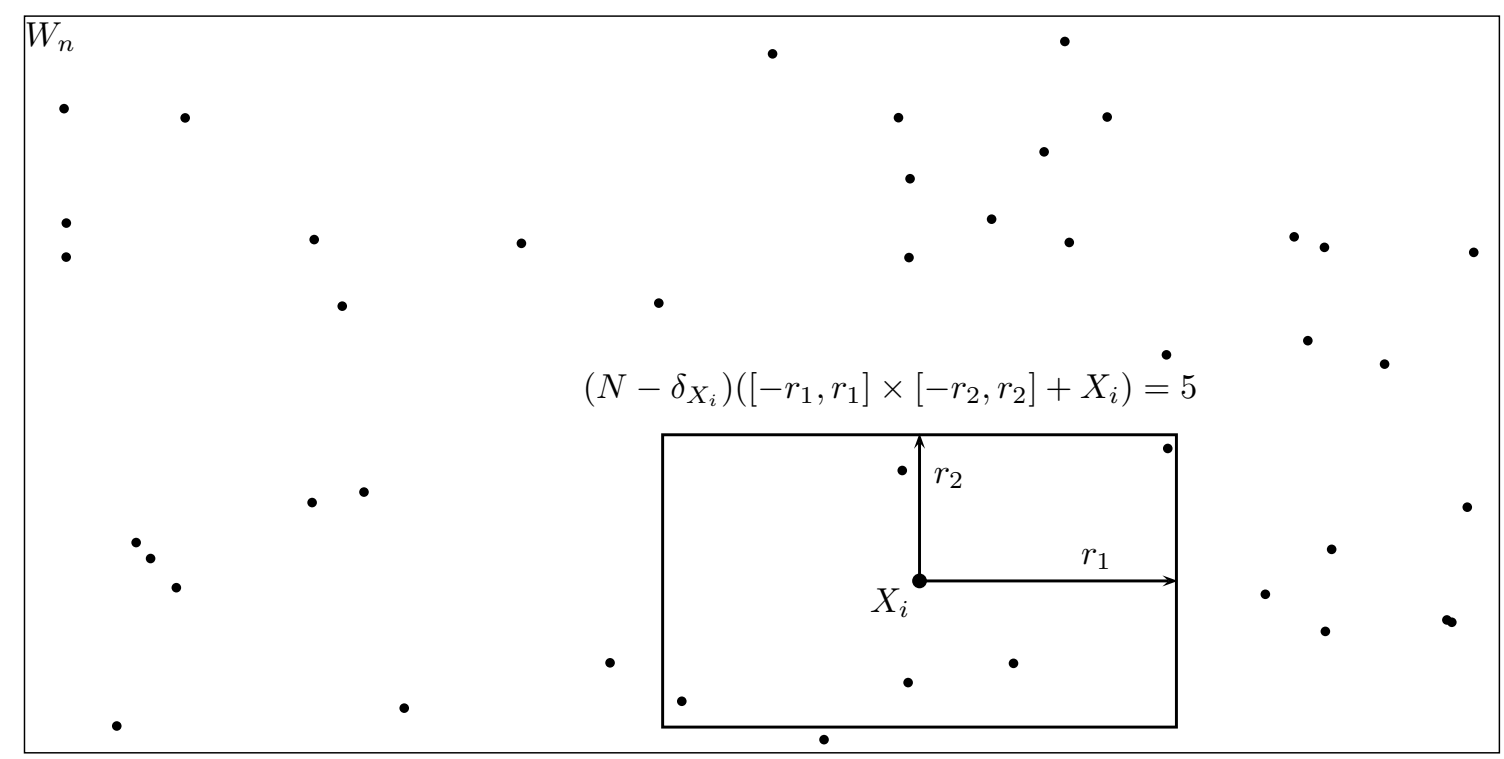

Fig.3: Computation of the empirical two-parameter K-function $\left.\widehat{\lambda^{2} K}\right)_{n, 1}\left(r_{1}, r_{2}\right)$ in $W_{n}$

Remark 1. To compute the d-parameter functions $\left(\widehat{\lambda^{2} K}\right)_{n, i}(\mathbf{r})$ on $I_{\varrho}$ we consider the point differences $X_{j}-X_{i} \in I_{\varrho}$ for $i \neq j$ and sort the differences in each coordinate (between 0 and $\varrho)$ according to increasing values generating multidimensional stair functions, see Fig. 1 for $d=2$. The use of the "naive" estimator (1.4) requires a reduction of $W_{n}$ to the smaller window $\left\{x \in W_{n}: B+x \subset W_{n}\right\}$ (minus-sampling) while the edge-corrected Horvitz-Thompson 
type estimator (1.5), e.g. see [8], exploits the full information of the point pattern contained in $W_{n}$ by weighting the influence of each pair $\left(X_{i}, X_{j}\right)$ for $i \neq j$. The unbiasedness of (1.5) has been first observed in [35], which is why (1.5) is also named Ohser-Stoyan estimator. In "large-domain statistics" the differences between (1.4), (1.5) and $\left.\widehat{\left(\lambda^{2} \mathcal{K}\right.}\right)_{n, 3}(B)$ are of minor importance, see Theorem 4.6 in [18] and Lemma 2 in Sect. 2.

The main aim of the present paper is to determine the weak functional limits of the centred and normed random processes $\left.\left\{\sqrt{\left|W_{n}\right|}\left(\widehat{\lambda^{2} K}\right)_{n, i}(\mathbf{r})-\mathbf{E}\left(\widehat{\lambda^{2} K}\right)_{n, i}(\mathbf{r})\right), \mathbf{r} \in I_{\varrho}\right\}$ for $i=1,2,3$ when $N$ is a stationary Poisson process on $\mathbb{R}^{d}$ with intensity $\lambda>0$ which, henceforth, will be denoted briefly by $N_{\lambda}$. It turns out that the cases of known and unknown intensity $\lambda>0$ have to be treated separately through different approaches leading to quite different multiparameter Gaussian limit processes.

To avoid ambiguity, we recall that $N_{\lambda}$ is defined as follows: (i) $N_{\lambda}(B)$ is Poisson distributed with mean $\lambda|B|$ for any $B \in \mathcal{B}_{b}^{d}$ and (ii) $N_{\lambda}\left(B_{1}\right), \ldots, N_{\lambda}\left(B_{k}\right)$ are mutually independent whenever $B_{1}, \ldots, B_{k} \in \mathcal{B}_{b}^{d}$ are pairwise disjoint. (i) and (ii) can be equivalently expressed as follows: For any $B \in \mathcal{B}_{b}^{d}$ with $|B|>0$, conditional on $N_{\lambda}(B)=k$, the random atoms $X_{1}, \ldots, X_{k}$ in $B$ are independently and uniformly distributed; the latter property is called "complete spatial randomness" (briefly CSR). Clearly, $N_{\lambda}$ is simple, stationary, ergodic and (ii) entails that the conditional expectation in (1.3) equals the unconditional mean $\mathbf{E} N(B)=\lambda|B|$ for $N=N_{\lambda}$. Thus, the $K$-measure coincides with the Lebesgue measure, i.e. $\mathcal{K}(\cdot)=|\cdot|$. Furthermore, ${\widehat{\left(\lambda^{2}\right)}}_{n}:=N_{\lambda}\left(W_{n}\right)\left(N_{\lambda}\left(W_{n}\right)-1\right) /\left|W_{n}\right|^{2}$ turns out to be an unbiased estimator for $\lambda^{2}$ which is seen by applying $(2.1)$ to $f\left(x_{1}, x_{2}\right)=\mathbf{1}_{W_{n}}\left(x_{1}\right) \mathbf{1}_{W_{n}}\left(x_{2}\right) /\left|W_{n}\right|^{2}$.

Using the above-defined estimators $\left(\widehat{\lambda^{2} \mathcal{K}}\right)_{n, i}(B), i=1,2,3$, for $N_{\lambda}=\sum_{i \geq 1} \delta_{X_{i}}$ we are now in a position to introduce the following six sequences of set-indexed mean zero random variables:

$$
Y_{n, i}(B)=\left(\widehat{\lambda^{2} \mathcal{K}}\right)_{n, i}(B)-\lambda^{2}|B| \quad \text { and } \quad Y_{n, 3}(B)=\left(\widehat{\lambda^{2} \mathcal{K}}\right)_{n, 3}(B)-\lambda^{2} J_{n}(B)
$$

for $i=1,2$ and $B \in \mathcal{B}_{b}^{d}$, where $J_{n}(B)=\left|W_{n}\right|^{-1} \int_{B}\left|W_{n} \cap\left(W_{n}-x\right)\right| \mathrm{d} x$, and likewise

$$
Z_{n, i}(B)=\left(\widehat{\lambda^{2} \mathcal{K}}\right)_{n, i}(B)-\widehat{\left(\lambda^{2}\right)_{n}}|B| \quad \text { and } \quad Z_{n, 3}(B)=\left(\widehat{\lambda^{2} \mathcal{K}}\right)_{n, 3}(B)-{\widehat{\left(\lambda^{2}\right.}}_{n} J_{n}(B)
$$

The set-indexed random processes in (1.8) resp. (1.9) define immediately the (multiparameter) random processes $Y_{n, i}(B(\mathbf{r})), \mathbf{r} \in I_{\varrho}$, and $Z_{n, i}(B(\mathbf{r})), \mathbf{r} \in I_{\varrho}$, for $i=1,2,3$. With a view to the Theorems 1 - 4 we recall the well-known fact that the distribution of mean zero multiparameter (resp. set-indexed) Gaussian process $\left\{G(\mathbf{r}), \mathbf{r} \in I_{\varrho}\right\}$ (resp. $\left\{G(B), B \in \mathcal{B}_{b}^{d}\right\}$ ) uniquely determined by its non-negative definite covariance function $\mathbf{E} G(\mathbf{s}) G(\mathbf{t}), \mathbf{s}, \mathbf{t} \in I_{\varrho}$, see [1] (resp. $\mathbf{E} G(B) G\left(B^{\prime}\right), B, B^{\prime} \in \mathcal{B}_{b}^{d}$, see [2]). For example, the standard set-indexed Wiener sheet $W(\cdot)$ on $\mathcal{B}_{b}^{d}$ is defined by $\mathbf{E} W(B)=0$ and $\mathbf{E} W(B) W\left(B^{\prime}\right)=\left|B \cap B^{\prime}\right|$, see [2] for details and a rigorous introduction. By putting $W(\mathbf{r}):=W(I(\mathbf{r}))$ with $I(\mathbf{r}):=\times_{i=1}^{d}\left[0, r_{i}\right]$ 
and $|\mathbf{r}|:=\prod_{i=1}^{d} r_{i}$ for $\mathbf{r}=\left(r_{1}, \ldots, r_{d}\right) \in \mathbb{R}_{+}^{d}$ we get the $d$-parameter (standard) Wiener sheet with covariance function $\mathbf{E} W(\mathbf{s}) W(\mathbf{t})=|I(\mathbf{s}) \cap I(\mathbf{t})|=|I(\mathbf{s} \wedge \mathbf{t})|=|\mathbf{s} \wedge \mathbf{t}|$ for $\mathbf{s}, \mathbf{t} \in \mathbb{R}_{+}^{d}$, where $\mathbf{s} \wedge(\vee) \mathbf{t}=\left(s_{1} \wedge(\vee) t_{1}, \ldots, s_{d} \wedge(\vee) t_{d}\right)$ for $\mathbf{s}=\left(s_{1}, \ldots, s_{d}\right), \mathbf{t}=\left(t_{1}, \ldots, t_{d}\right) \in \mathbb{R}_{+}^{d}$. This implies $W(B(\mathbf{r})) \stackrel{\mathbf{d}}{=} 2^{d / 2} W(\mathbf{r})$ and $W\left(I_{\varrho}\right) \stackrel{\mathbf{d}}{=} \varrho^{d / 2} W\left(I_{1}\right)$.

Finally, we recall that $\boldsymbol{D}[0, \varrho]^{d}$ consists of all real-valued functions $f$ on the cube $I_{\varrho}$ which are "continuous from above" and have "limits from below" in each $\mathbf{r} \in I_{\varrho}$, see [3], [34] for a precise definition and the concept of weak convergence in the Skorokhod-space $\boldsymbol{D}[0, \varrho]^{d}$.

Theorem 1. The random processes $\left\{Y_{n, i}(\mathbf{r}):=\sqrt{\left|W_{n}\right|} Y_{n, i}(B(\mathbf{r})): \mathbf{r} \in I_{\varrho}\right\}$ defined in (1.8) and driven by $N_{\lambda}=\sum_{i \geq 1} \delta_{X_{i}}$ belong a.s. to the Skorokhod-space $\boldsymbol{D}[0, \varrho]^{d}$ for $i=1,2,3$ and $n \in \mathbb{N}$. The weak convergence

$$
\left\{Y_{n, i}(\mathbf{r}): \mathbf{r} \in I_{\varrho}\right\} \underset{n \rightarrow \infty}{\stackrel{\mathbf{d}}{\longrightarrow}}\left\{Y(\mathbf{r}): \mathbf{r} \in I_{\varrho}\right\}
$$

holds in $\boldsymbol{D}[0, \varrho]^{d}$, where $\left\{Y(\mathbf{r}): \mathbf{r} \in I_{\varrho}\right\}$ is an a.s. continuous Gaussian process with mean zero and covariance function $\mathbf{E} Y(\mathbf{s}) Y(\mathbf{t})=\lambda^{2} 2^{d+1}|\mathbf{s} \wedge \mathbf{t}|\left(1+\lambda 2^{d+1}|\mathbf{s} \vee \mathbf{t}|\right)$.

Moreover, $\left\{Y(\mathbf{r}): \mathbf{r} \in I_{\varrho}\right\} \stackrel{\mathbf{d}}{=}\left\{\lambda 2^{(d+1) / 2}\left(W(\mathbf{r})+\left(\sqrt{1+2^{d+1} \lambda \varrho^{d}}-1\right) \varrho^{-d}|\mathbf{r}| W(\varrho)\right): \mathbf{r} \in I_{\varrho}\right\}$, where $\varrho:=(\varrho, \ldots, \varrho)$ and $\stackrel{\mathrm{d}}{=}$ indicates "equality in distribution".

The counterpart of (1.10) when the intensity $\lambda$ is unknown and $\lambda^{2}$ is replaced by $\widehat{\left(\lambda^{2}\right)_{n}}$ is the subject of

Theorem 2. The random processes $\left\{Z_{n, i}(\mathbf{r}):=\sqrt{\left|W_{n}\right|} Z_{n, i}(B(\mathbf{r})): \mathbf{r} \in I_{\varrho}\right\}$ defined in (1.9) and driven by $N_{\lambda}=\sum_{i \geq 1} \delta_{X_{i}}$ belong a.s. to the Skorokhod-space $\boldsymbol{D}[0, \varrho]^{d}$ for $i=1,2,3$ and $n \in \mathbb{N}$. The weak convergence

$$
\left\{Z_{n, i}(\mathbf{r}): \mathbf{r} \in I_{\varrho}\right\} \underset{n \rightarrow \infty}{\stackrel{\mathbf{d}}{\longrightarrow}}\left\{Z(\mathbf{r}): \mathbf{r} \in I_{\varrho}\right\} \stackrel{\mathbf{d}}{=}\left\{\lambda 2^{(d+1) / 2} W(\mathbf{r}): \mathbf{r} \in I_{\varrho}\right\}
$$

holds in $\boldsymbol{D}[0, \varrho]^{d}$.

Remark 2. Theorem 1 and 2 generalize two functional limit theorems in [16] proven for empirical processes associated with Ripley's $K$-function $\{K(r), 0 \leq r \leq \varrho\}$. Central limit theorems (short: CLT's) for empirical functionals related with (1.1) in case of non-Poissonian point processes can be found e.g. in [25], [26], [15], [28].

The rest of the paper is organized as follows: In Sect. 2 we put together some lemmas which facilitate the proofs of Theorem 1 and 2 given in Sect. 3 and 4, respectively. Further, we prove the tightness of the random processes occurring in (1.10) and (1.11). In both Sect. 3 and 4 we formulate CLT's which extend the weak convergence of the finite-dimensional distributions (short: FIDI's) in Theorem 1 and 2. Both Theorem 3 and 4 seem to be of interest in its own right. In the final Sect. 5 we study the supremum as well as the integral of the squared Gaussian limit processes over the cube $I_{\varrho}$. These results enable us to establish asymptotic 
goodness-of-fit tests in the sense of Kolmogorov-Smirnov as well as Cramér-von Mises for the multiparameter $K$-function of stationary Poisson processes on $\mathbb{R}^{d}$. For $d=2$ a table of relevant quantiles of the test statistics (in case of unknown intensity) has been determined via simulation of the Wiener sheet on $[0,1]^{2}$. This provides a test for checking the CSR hypothesis of planar point patterns. Throughout, let $c_{1}, c_{2}, \ldots$ denote positive constants not depending on $n$.

\section{Preliminary Results}

In this section we prove some auxiliary results (all of them apply only for $N_{\lambda}$ ) which among others show that the limits of the processes $Y_{n, i}(\cdot)$ ( resp. $\left.Z_{n, i}(\cdot)\right)$ (if they exist!) are same for each $i=1,2,3$. Furthermore, it will be shown that all of these sequences of random processes are tight in $\boldsymbol{D}[0, \varrho]^{d}$. To begin with we state two basic relations for $N_{\lambda}$.

Lemma 1. For any integrable function $f \mid\left(\mathbb{R}^{d}\right)^{k} \mapsto \mathbb{R}^{1}$, i.e. $f \in \boldsymbol{L}^{\mathbf{1}}\left(\left(\mathbb{R}^{d}\right)^{k}\right)$, for some $k \in \mathbb{N}$ and any $f_{1}, f_{2} \in \boldsymbol{L}^{\mathbf{1}}\left(\left(\mathbb{R}^{d}\right)^{2}\right) \cap \boldsymbol{L}^{\mathbf{2}}\left(\left(\mathbb{R}^{d}\right)^{2}\right)$,

$$
\begin{gathered}
\mathbf{E}\left(\sum_{i_{1}, \ldots, i_{k} \geq 1}^{\neq} f\left(X_{i_{1}}, \ldots, X_{i_{k}}\right)\right)=\lambda^{k} \int \cdots \int f\left(x_{1}, \ldots, x_{k}\right) \mathrm{d} x_{1} \cdots \mathrm{d} x_{k}, \\
\operatorname{Cov}\left(\sum_{i, j \geq 1}^{\neq} f_{1}\left(X_{i}, X_{j}\right), \sum_{i, j \geq 1}^{\neq} f_{2}\left(X_{i}, X_{j}\right)\right)=\lambda^{2} \iint f_{1}(x, y)\left(f_{2}(x, y)+f_{2}(y, x)\right) \mathrm{d} x \mathrm{~d} y \\
+\lambda^{3} \iiint f_{1}(x, y)\left(f_{2}(x, z)+f_{2}(z, x)+f_{2}(y, z)+f_{2}(z, y)\right) \mathrm{d} x \mathrm{~d} y \mathrm{~d} z,
\end{gathered}
$$

where $\int$ is written shorthand for an integral over $\mathbb{R}^{d}$.

Proof of Lemma 1. To prove (2.1) we remember the definition of the kth-order factorial moment measure and its specific shape for Poisson processes, see [10], p. 72. The proof of (2.1) is accomplished by applying the (ordinary) Campbell theorem to multiple sums of $k$-tuples $\left(X_{i_{1}}, \ldots, X_{i_{k}}\right)$ of pairwise distinct atoms of $N_{\lambda}$, see [9], [8]. To verify the second assertion we first rewrite the product $S_{1} S_{2}$, where $S_{a}=\sum^{\neq}{ }_{i, j \geq 1} f_{a}\left(X_{i}, X_{j}\right)$ for $a=1,2$, as sums over pairs $\left(X_{i}, X_{j}\right)$, triples $\left(X_{i}, X_{j}, X_{k}\right)$ and quadruples $\left(X_{i}, X_{j}, X_{k}, X_{\ell}\right)$ of pairwise distinct atoms of $N_{\lambda}$. Taking the expectation of these seven sums calculated according to (2.1) reveals that the expectation over the sum of quadruples is just equal to $\mathbf{E} S_{1} \mathbf{E} S_{2}$. For general fourth-order stationary point processes a corresponding formula in terms of factorial moment and cumulant measures is stated in [15], p. 97. Hence, the sum of the remaining two expectations of pairs and four expectations over triples of atoms of $N_{\lambda}$ equals $\operatorname{Cov}\left(S_{1}, S_{2}\right)$. Using again (2.1) with an appropriate choice of $f$ for $k=2$ and $k=3$, respectively, leads immediately to (2.3) and completes the proof of Lemma 1.

To be somewhat more general we replace the set-indexed estimators $\left(\widehat{\lambda^{2} \mathcal{K}}\right)_{n, i}(B)$ defined in Sect. 1 by function-indexed empirical processes estimating the functional $\lambda^{2} \int g(x) \mathrm{d} x$. for 
any real-valued function $g \in \boldsymbol{L}_{\mathbf{1}}\left(\mathbb{R}^{d}\right) \cap \boldsymbol{L}_{\mathbf{2}}\left(\mathbb{R}^{d}\right)$. Substituting the indicator function $\mathbf{1}_{B}$ in (1.1), (1.4), (1.5) and (1.6) by $g$ we get following mean zero function-indexed empirical processes

$$
\begin{aligned}
Y_{n, 1}(g) & :=\frac{1}{\left|W_{n}\right|} \sum_{i, j \geq 1}^{\neq} \mathbf{1}_{W_{n}}\left(X_{i}\right) g\left(X_{j}-X_{i}\right)-\lambda^{2} J(g), \\
Y_{n, 2}(g) & :=\sum_{i, j \geq 1}^{\neq} \frac{\mathbf{1}_{W_{n}}\left(X_{i}\right) \mathbf{1}_{W_{n}}\left(X_{j}\right) g\left(X_{j}-X_{i}\right)}{\left|\left(W_{n}-X_{i}\right) \cap\left(W_{n}-X_{j}\right)\right|}-\lambda^{2} J(g), \\
Y_{n, 3}(g) & :=\frac{1}{\left|W_{n}\right|} \sum_{i, j \geq 1}^{\neq} \mathbf{1}_{W_{n}}\left(X_{i}\right) \mathbf{1}_{W_{n}}\left(X_{j}\right) g\left(X_{j}-X_{i}\right)-\lambda^{2} J_{n}(g)
\end{aligned}
$$

and, for $i=1,2$,

$$
\begin{aligned}
& Z_{n, i}(g):=Y_{n, i}(g)+\left(\lambda^{2}-{\widehat{\left(\lambda^{2}\right)_{n}}}\right) J(g) \quad \text { and } \quad Z_{n, 3}(g):=Y_{n, 3}(g)+\left(\lambda^{2}-{\widehat{\left(\lambda^{2}\right)_{n}}}\right) J_{n}(g), \\
& \text { where } \quad J_{n}(g):=\left|W_{n}\right|^{-1} \int g(x)\left|W_{n} \cap\left(W_{n}-x\right)\right| \mathrm{d} x \underset{n \rightarrow \infty}{\longrightarrow} J(g):=\int g(x) \mathrm{d} x .
\end{aligned}
$$

The sequences $Y_{n, i}(g)$ and $Z_{n, i}(g)$ blown up with $\sqrt{\left|W_{n}\right|}$ will presumably converge in distribution to Gaussian limits. The next lemma guarantees that, by virtue of Slutsky's lemma, see [38], these limits does not depend on $i=1,2,3$.

Lemma 2. The following limits hold for $i=1,3$ if $g, h \in \boldsymbol{L}^{\mathbf{1}}\left(\mathbb{R}^{d}\right) \cap \boldsymbol{L}^{\mathbf{2}}\left(\mathbb{R}^{d}\right)$, and for $i=2$ if $g, h$ are boundedly supported and square integrable over $\mathbb{R}^{d}$ :

$$
\begin{aligned}
& \left|W_{n}\right| \mathbf{E}\left(Y_{n, i}(g)-Y_{n, 3}(g)\right)^{2} \underset{n \rightarrow \infty}{\longrightarrow} 0 \quad \text { and } \quad\left|W_{n}\right| \mathbf{E}\left(Z_{n, i}(g)-Z_{n, 3}(g)\right)^{2} \underset{n \rightarrow \infty}{\longrightarrow} 0, \\
& \lim _{n \rightarrow \infty}\left|W_{n}\right| \mathbf{E}\left(Y_{n, i}(g) Y_{n, i}(h)\right)=\lambda^{2} \int g(x)(h(x)+h(-x)) \mathrm{d} x+4 \lambda^{3} J(g) J(h), \\
& \lim _{n \rightarrow \infty}\left|W_{n}\right| \mathbf{E}\left(Z_{n, i}(g) Z_{n, i}(h)\right)=\lambda^{2} \int g(x)(h(x)+h(-x)) \mathrm{d} x .
\end{aligned}
$$

Proof of Lemma 2. From (2.7) we get the identity

$$
Z_{n, i}(g)-Z_{n, 3}(g)=Y_{n, i}(g)-Y_{n, 3}(g)+\left(\lambda^{2}-\widehat{\left(\lambda^{2}\right)_{n}}\right)\left(J(g)-J_{n}(g)\right)
$$

and the inequality $\mathbf{E}(X+Y)^{2} \leq 2 \mathbf{E} X^{2}+2 \mathbf{E} Y^{2}$ yields

$$
\left|W_{n}\right| \mathbf{E}\left(Z_{n, i}(g)-Z_{n, 3}(g)\right)^{2} \leq 2\left|W_{n}\right| \mathbf{E}\left(Y_{n, i}(g)-Y_{n, 3}(g)\right)^{2}+2\left|W_{n}\right| \operatorname{Var}\left(\widehat{\left(\lambda^{2}\right)_{n}}\right)\left(J(g)-J_{n}(g)\right)^{2}
$$

Employing (2.3) with $f_{1}(x, y)=f_{2}(x, y)=\mathbf{1}_{W_{n}}(x) \mathbf{1}_{W_{n}}(y) /\left|W_{n}\right|^{2}$ gives $\left|W_{n}\right| \operatorname{Var}\left(\widehat{\left(\lambda^{2}\right)_{n}}\right)=$ $4 \lambda^{3}+2 \lambda^{2} /\left|W_{n}\right|$. Since $J_{n}(g) \underset{n \rightarrow \infty}{\longrightarrow} J(g)$, the second assertion of (2.8) follows from the first one. To show the first assertion of (2.8) we first treat the case $i=1$. Here and later in this proof we use the abbreviations $R_{n}(x)=\left|W_{n} \cap\left(W_{n}-x\right)\right| /\left|W_{n}\right|$ and $R_{n}(x, y)=\mid W_{n} \cap\left(W_{n}-x\right) \cap$ $\left(W_{n}-y\right)|/| W_{n} \mid$. The properties the CAS $\left(W_{n}\right)$ guarantee that $1 \geq R_{n}(x) \geq R_{n}(x, y) \underset{n \rightarrow \infty}{\longrightarrow} 1$ 
for any fixed $x, y \in \mathbb{R}^{d}$. For $f_{1}(x, y)=f_{2}(x, y)=\mathbf{1}_{W_{n}}(x) \mathbf{1}_{W_{n}^{c}}(y) g(y-x) /\left|W_{n}\right|$ the formula (2.3) yields after some rearrangements that

$$
\begin{aligned}
& \left|W_{n}\right| \mathbf{E}\left(Y_{n, 1}(g)-Y_{n, 3}(g)\right)^{2}=\lambda^{2} \int\left(1-R_{n}(z)\right) g^{2}(z) \mathrm{d} z \\
+ & \lambda^{3} \iint\left[1-R_{n}(y)-R_{n}(z)+R_{n}(y, z)+R_{n}(z-y)-R_{n}(-y,-z)\right] g(y) g(z) \mathrm{d} y \mathrm{~d} z,
\end{aligned}
$$

whence it follows the first part (2.8) for $i=1$ by Lebesgue's dominated convergence theorem. To show (2.8) for $i=2$ we assume that $g(x)=0$ for $x \notin B(\mathbf{o}, \varrho)$ for some $\varrho>0$. As before we use (2.3) for $\left.f_{1}(x, y)=f_{2}(x, y)=\mathbf{1}_{W_{n}}(x) \mathbf{1}_{W_{n}}(y) g(y-x)\left(1-R_{n}(y-x)\right) / \mid\left(W_{n}-x\right) \cap\left(W_{n}-y\right)\right) \mid$ and arrive at

$$
\begin{aligned}
& \left|W_{n}\right| \mathbf{E}\left(Y_{n, 2}(g)-Y_{n, 3}(g)\right)^{2}=\lambda^{2} \int_{B(\mathbf{o}, \varrho)} \frac{\left(1-R_{n}(z)\right)^{2}}{R_{n}(z)} g(z)(g(z)+g(-z)) \mathrm{d} z \\
+ & \lambda^{3} \int_{B(\mathbf{o}, \varrho)} \int_{B(\mathbf{o}, \varrho)} R_{n}(y, z) \frac{1-R_{n}(y)}{R_{n}(y)} \frac{1-R_{n}(z)}{R_{n}(z)}(g(y)+g(-y))(g(z)+g(-z)) \mathrm{d} y \mathrm{~d} z .
\end{aligned}
$$

The convexity of $W_{n}$ yields $\left|W_{n}\right|-\left|W_{n} \cap\left(W_{n}-x\right)\right|=\left|W_{n} \cap\left(W_{n}^{c}-x\right)\right| \leq \varrho\left|\partial W_{n}\right|_{d-1}$ for $x \in B(\mathbf{o}, \varrho)$ so that combined with $(1.2)$,

$$
\sup _{x \in B(\mathbf{o}, \varrho)}\left(\frac{1}{R_{n}(x)}-1\right) \leq \frac{d \varrho}{r\left(W_{n}\right)-d \varrho} \underset{n \rightarrow \infty}{\longrightarrow} 0
$$

The latter relation implies $\left|W_{n}\right| \mathbf{E}\left(Y_{n, 2}(g)-Y_{n, 3}(g)\right)^{2} \underset{n \rightarrow \infty}{\longrightarrow} 0$ completing the proof of (2.8).

For $i=3$ the relations (2.9) and (2.10) can be easily deduced from the identities

$$
\begin{aligned}
\left|W_{n}\right| \mathbf{E}\left(Y_{n, 3}(g) Y_{n, 3}(h)\right) & =\lambda^{2} \int g(x)(h(x)+h(-x)) R_{n}(x) \mathrm{d} y \\
& +\lambda^{3} \iint(g(x)+g(-x))(h(y)+h(-y)) R_{n}(x, y) \mathrm{d} x \mathrm{~d} y
\end{aligned}
$$

and

$$
\begin{array}{r}
\left|W_{n}\right| \mathbf{E}\left(Z_{n, 3}(g), Z_{n, 3}(h)\right)=\lambda^{2} \int g(x)(h(x)+h(-x)) R_{n}(x) \mathrm{d} y-\frac{2 \lambda^{2}}{\left|W_{n}\right|} J_{n}(g) J_{n}(h) \\
+\lambda^{3} \iint(g(x)+g(-x))(h(y)+h(-y)) R_{n}(x, y) \mathrm{d} x \mathrm{~d} y-4 \lambda^{3} J_{n}(g) J_{n}(h)
\end{array}
$$

by the integrability assumptions on $g$ and $h$ combined with $R_{n}(y) \underset{n \rightarrow \infty}{\longrightarrow} 1$ and $R_{n}(x, y) \underset{n \rightarrow \infty}{\longrightarrow} 1$ for any fixed $x, y \in \mathbb{R}^{d}$. By the same arguments, without calculating the expressions of $\left|W_{n}\right| \mathbf{E}\left(Y_{n, 1}(g) Y_{n, 1}(h)\right)$ and $\left|W_{n}\right| \mathbf{E}\left(Z_{n, 1}(g) Z_{n, 1}(h)\right)$ in detail, we can confirm (2.9) and (2.10) for $i=1$. On the other hand, in the remaining case $i=2$ the integral representations of $\left|W_{n}\right| \mathbf{E}\left(Y_{n, 2}(g) Y_{n, 2}(h)\right)$ and $\left|W_{n}\right| \mathbf{E}\left(Z_{n, 2}(g) Z_{n, 2}(h)\right)$ contain the reciprocals $1 / R_{n}(x)$ and $1 / R_{n}(y)$ which, by (2.13), converge uniformly to 1 for $x, y \in B(\mathbf{o}, \varrho)$. This provides the asymptotic covariances (2.9) as well as (2.10) for $i=2$ and completes the proof of Lemma 2 . 
Corollary 1. For indicator functions $g=\mathbf{1}_{A}, h=\mathbf{1}_{B}$ of any $A, B \in \mathcal{B}_{b}^{d}$ the asymptotic covariances (2.9) and (2.10) take the form

$$
\begin{aligned}
\lim _{n \rightarrow \infty}\left|W_{n}\right| \mathbf{E} Y_{n, i}(A) Y_{n, i}(B) & =\lambda^{2}|A \cap B|+\lambda^{2}|A \cap(-B)|+4 \lambda^{3}|A||B| \\
\lim _{n \rightarrow \infty}\left|W_{n}\right| \mathbf{E} Z_{n, i}(A) Z_{n, i}(B) & =\lambda^{2}|A \cap B|+\lambda^{2}|A \cap(-B)| .
\end{aligned}
$$

Remark 3. The asymptotic variances of $\sqrt{\left|W_{n}\right|} Y_{n, i}(B)$ and $\sqrt{\left|W_{n}\right|} Z_{n, i}(B)$ become minimal (resp. maximal) for sets $B \subset \mathcal{B}_{b}^{d}$ satisfying $|B \cap(-B)|=0 \quad($ resp. $|B \cap(-B)|=|B|)$.

In order to simplify the proofs of the Theorems 3 and 4 we need the following "truncation lemma". For this purpose we assign to each function $g \mid \mathbb{R}^{d} \mapsto \mathbb{R}^{1}$ the truncated function $g_{a, \varrho}(x)=g(x)$, if $x \in C_{\varrho}$ and $|g(x)| \leq a$, and $g(x)=0$ otherwise, where $a, \varrho>0$ are suitably chosen.

Lemma 3. For any real-valued function $g \in \boldsymbol{L}^{\mathbf{1}}\left(\mathbb{R}^{d}\right) \cap \boldsymbol{L}^{\mathbf{2}}\left(\mathbb{R}^{d}\right)$ the following estimates hold:

$$
\sup _{n \in \mathbb{N}}\left|W_{n}\right| \mathbf{E}\left(Y_{n, 1}(g)-Y_{n, 1}\left(g_{a, \varrho}\right)\right)^{2} \leq 2 \lambda^{2} c_{2}(a, \varrho)+4 \lambda^{3} c_{1}(a, \varrho)^{2}
$$

and

$$
\sup _{n \in \mathbb{N}:\left|W_{n}\right| \geq 1}\left|W_{n}\right| \mathbf{E}\left(Z_{n, 3}(g)-Z_{n, 3}\left(g_{a, \varrho}\right)\right)^{2} \leq 2 \lambda^{2} c_{2}(a, \varrho)+\left(2 \lambda^{2}+8 \lambda^{3}\right) c_{1}(a, \varrho)^{2},
$$

where $c_{j}(a, \varrho):=\int|g(x)|^{j} \mathbf{1}(|g(x)|>a) \mathrm{d} x \wedge \int|g(x)|^{j} \mathbf{1}\left(x \notin C_{\varrho}\right) \mathrm{d} x$ for $j=1,2$.

Proof of Lemma 3. Since $Y_{n, 1}(g)-Y_{n, 1}\left(g_{a, \varrho}\right)=Y_{n, 1}\left(g-g_{a, \varrho}\right)$ the relation

$$
\left|W_{n}\right| \mathbf{E}\left(Y_{n, 1}(h)\right)^{2}=\lambda^{2} \int h(x)\left(h(x)+R_{n}(x) h(-x)\right) \mathrm{d} x+\lambda^{3} J(h)\left(J(h)+3 J_{n}(h)\right),
$$

which can be obtained in like manner as (2.14), implies for $h=g-g_{a, \varrho}$ that

$$
\sup _{n \in \mathbb{N}}\left|W_{n}\right| \mathbf{E}\left(Y_{n, 1}(g)-Y_{n, 1}\left(g_{a, \varrho}\right)\right)^{2} \leq 2 \lambda^{2} J\left(\left(g-g_{a, \varrho}\right)^{2}\right)+4 \lambda^{3} J\left(\left|g-g_{a, \varrho}\right|\right)^{2} .
$$

Together with $J\left(\left|g-g_{a, \varrho}\right|^{j}\right) \leq c_{j}(a, \varrho)$ for $j=1,2$ we arrive at $(2.16)$.

Using (2.15) directly with $h=g=g-g_{a, \varrho}$ we get in the same way that

$$
\sup _{n \in \mathbb{N}:\left|W_{n}\right| \geq 1}\left|W_{n}\right| \mathbf{E}\left(Z_{n, 3}(g)-Z_{n, 3}\left(g_{a, \varrho}\right)\right)^{2} \leq 2 \lambda^{2} J\left(\left(g-g_{a, \varrho}\right)^{2}\right)+\left(2 \lambda^{2}+8 \lambda^{3}\right) J\left(\left|g-g_{a, \varrho}\right|\right)^{2},
$$

which gives (2.17).

Remark 4. Since $c_{1}(a, \varrho) \vee c_{2}(a, \varrho) \longrightarrow 0$ as $a \rightarrow \infty$ or $\varrho \rightarrow \infty$ Slutsky's theorem, see [38], says that in order to prove the weak limits of the sequences $\sqrt{\left|W_{n}\right|} Y_{n, i}(g)$ and $\sqrt{\left|W_{n}\right|} Z_{n, i}(g)$ for $g \in \boldsymbol{L}^{\mathbf{1}}\left(\mathbb{R}^{d}\right) \cap \boldsymbol{L}^{\mathbf{2}}\left(\mathbb{R}^{d}\right)$ it suffices to verify these limits only for some bounded and boundedly supported $g$. 
Lemma 4. For sufficiently large $n \in \mathbb{N}, i=1,2,3$ and any disjoint sets $A, B \in \mathcal{B}_{b}^{d} \cap C_{\varrho}$ the set-indexed processes (1.8) and (1.9) fulfil the estimates

$\left|W_{n}\right| \mathbf{E}\left(Y_{n, i}(A) Y_{n, i}(B)\right)^{2} \leq c_{1}(\varrho)|A||B| \quad$ and $\quad\left|W_{n}\right| \mathbf{E}\left(Z_{n, i}(A) Z_{n, i}(B)\right)^{2} \leq c_{2}(\varrho)|A||B|$.

For any $n \in \mathbb{N}$ and each $i=1,2,3$ the multiparameter processes

$\left\{Y_{n, i}(\mathbf{r}):=\sqrt{\left|W_{n}\right|} Y_{n, i}(B(\mathbf{r})), r \in I_{\varrho}\right\}$ and $\left\{Z_{n, i}(\mathbf{r}):=\sqrt{\left|W_{n}\right|} Z_{n, i}(B(\mathbf{r})), r \in I_{\varrho}\right\}$ belong a.s. to $\boldsymbol{D}[0, \varrho]^{d}$. Moreover, both sequence $\left\{Y_{n, i}(\mathbf{r}): \mathbf{r} \in I_{\varrho}\right\}$ and $\left\{Z_{n, i}(\mathbf{r}): \mathbf{r} \in I_{\varrho}\right\}$ are tight in $\boldsymbol{D}[0, \varrho]^{d}$.

Proof of Lemma 4. The calculation of the mixed fourth-order moment $\mathbf{E}\left(Y_{n, i}(A) Y_{n}, i(B)\right)^{2}$ starts with the representation of the product $Y_{n, i}(A) Y_{n}, i(B)$ through sums of multiple sums over $k$-tuples $\left(X_{i_{1}}, \ldots, X_{i_{k}}\right)$ of pairwise distinct atoms of $N_{\lambda}$ for $k=2,3,4$. After simplifying these multiple sums due to $A \cap B=\emptyset$, we square the whole sum of terms and evaluate the expectations by applying repeatedly the Campbell-type formula $(2.1)$ for $k \in\{2,3, \ldots, 8\}$. The details of these rather lengthy and tedious, but straightforward calculations proving lastly both estimates of Lemma 4 are left to the reader.

For $i=1,2,3$ each realization of the random process $Y_{n, i}(\mathbf{r})=\sqrt{\left|W_{n}\right|} Y_{n, i}(B(\mathbf{r}))$ on the cube $I_{\varrho}$ can be written as scaled difference of the step function $\left.\widehat{\lambda^{2} \mathcal{K}}\right)_{n, i}(B(\mathbf{r}))$ having at most $X_{n}\left(C_{\varrho}\right)$ jumps on each coordinate-axis and the continuous function $\lambda^{2}|B(\mathbf{r})|$ (for $i=1,2$ ) or $\lambda^{2} \int_{B(\mathbf{r})} R_{n}(x) \mathrm{d} x$ (for $i=3$ ). Note that step functions are finite linear combinations of indicator functions $\mathbf{r}=\left(r_{1}, \ldots, r_{d}\right) \mapsto \prod_{i=1}^{d} \mathbf{1}_{J_{i}}\left(r_{i}\right)$ for $J_{i}=\left[a_{i}, b_{i}\right), 0 \leq a_{i}<b_{i} \leq \varrho$ or $J_{i}=\{\varrho\}, i=1, \ldots, d$. All these step functions and continuous functions belong to $\boldsymbol{D}[0, \varrho]^{d}$, see [3], p. 1662. Since $N_{\lambda}=\sum_{i \geq 1} \delta_{X_{i}}$ is a locally finite counting measure we have $\mathbf{P}\left(X_{n}\left(C_{\varrho}\right)<\right.$ $\infty)=1$ yielding that $\mathbf{P}\left(\left\{Y_{n, i}(\mathbf{r}): \mathbf{r} \in I_{\varrho}\right\} \in \boldsymbol{D}[0, \varrho]^{d}\right)=1$. (2.7) reveals that the same applies for $\left\{Z_{n, i}(\mathbf{r}): \mathbf{r} \in I_{\varrho}\right\}$.

To prove the tightness of the sequence $Y_{n, i}(\mathbf{r})=\sqrt{\left|W_{n}\right|} Y_{n, i}(B(\mathbf{r})), r \in I_{\varrho}$, in $\boldsymbol{D}[0, \varrho]^{d}$ we employ the following moment criterion given in [3], p. 1658, see also [4] for $d=1$ : For any two adjacent half-open hyper-rectangles $B_{p}=\times_{i \neq p}\left(s_{i}, t_{i}\right] \times\left(s_{p}, t_{p}\right]$ and $B_{p}^{\prime}=\times_{i \neq p}\left(s_{i}, t_{i}\right] \times\left(t_{p}, t_{p}^{\prime}\right]$ $\left(s_{p}<t_{p}<t_{p}^{\prime}\right)$ in $I_{\varrho}$ with a common $p$ th face for some $p \in\{1, \ldots, d\}$ we suppose that

$$
\mathbf{E}\left|\widetilde{Y}_{n, i}\left(B_{p}\right) \widetilde{Y}_{n, i}\left(B_{p}^{\prime}\right)\right|^{\gamma} \leq\left(\nu(B) \nu\left(B^{\prime}\right)\right)^{\beta}
$$

for fixed $\gamma>0, \beta>1 / 2$ and some finite measure $\nu($.$) on I_{\varrho}$, where $\widetilde{Y}_{n, i}\left(B_{p}\right)$ is the increment of d-parameter process $Y_{n, i}(\mathbf{r})$ around the hyper-rectangle $B_{p}$. It is easily checked that $\widetilde{Y}_{n, i}\left(B_{p}\right)=\sqrt{\left|W_{n}\right|} Y_{n, i}\left(\cup_{\varepsilon_{1}, \ldots, \varepsilon_{d} \in\{-1,1\}} B_{p}\left(\varepsilon_{1}, \ldots, \varepsilon_{d}\right)\right)$, where $B_{p}\left(\varepsilon_{1}, \ldots, \varepsilon_{d}\right)$ is obtained from $B_{p}$ by multiplying the coordinates of each vertex of $B_{p}$ by $\varepsilon_{1}, \ldots, \varepsilon_{d}$. By doing the same for $B_{p}^{\prime}$ we see that the corresponding union of the sets $B_{p}^{\prime}\left(\varepsilon_{1}, \ldots, \varepsilon_{d}\right)$ has no common point with the other union set. Hence, by the first estimate of Lemma 4 we can easily deduce the estimate

$$
\left|W_{n}\right| \mathbf{E}\left(\widetilde{Y}_{n, i}\left(B_{p}\right) \widetilde{Y}_{n, i}\left(B_{p}^{\prime}\right)\right)^{2} \leq c_{1}(\varrho) 4^{d}\left|B_{p}\right|\left|B_{p}^{\prime}\right|
$$


proving (2.19) for $\gamma=2, \beta=1$ and $\nu(\cdot)=|\cdot|$. In view of (2.7) and the second estimate of Lemma 4 we get an analogous estimate for the increments of the processes $\left\{Z_{n, i}(\mathbf{r})=\right.$ $\left.\sqrt{\left|W_{n}\right|} Z_{n, i}(B(\mathbf{r})), r \in I_{\varrho}\right\}$ with a possibly different constant on the r.h.s. of (2.20). Thus, all assertions of Lemma 4 are completely proved.

\section{CLT FOR THE SEQUENCES $\sqrt{\left|W_{n}\right|} \boldsymbol{Y}_{n, i}(g)$ AND PROOF OF THEOREM 1}

We shall prove asymptotic normality for each of the sequences (2.4), (2.5) and (2.6) being defined for a stationary Poisson process $N_{\lambda}$ with intensity $\lambda>0$ and some CAS $\left(W_{n}\right)$ in $\mathbb{R}^{d}$. Further, let $\mathcal{N}\left(\mu, \sigma^{2}\right)$ denote a Gaussian random variable with mean $\mu$ and variance $\sigma^{2}>0$,

and $\underset{n \rightarrow \infty}{\stackrel{\mathbf{d}}{\longrightarrow}}$ indicates convergence in distribution or weak convergence of the random elements under consideration.

Theorem 3. For any $g \in \boldsymbol{L}_{\mathbf{1}}\left(\mathbb{R}^{d}\right) \cap \boldsymbol{L}_{\mathbf{2}}\left(\mathbb{R}^{d}\right)$ and $i=1$ or $i=3$,

$$
\begin{gathered}
\sqrt{\left|W_{n}\right|} Y_{n, i}(g) \underset{n \rightarrow \infty}{\stackrel{\mathbf{d}}{\longrightarrow}} \mathcal{N}\left(0, \sigma^{2}(g)\right) \quad \text { with } \\
\sigma^{2}(g)=\lambda^{2} \int g(x)(g(x)+g(-x)) \mathrm{d} x+4 \lambda^{3} J(g)^{2}, \quad \text { where } J(g)=\int g(x) \mathrm{d} x .
\end{gathered}
$$

Furthermore, (3.1) holds for $i=2$, if $g \in \boldsymbol{L}_{\mathbf{2}}\left(\mathbb{R}^{d}\right)$ and $\operatorname{supp}(g):=\{x: g(x) \neq 0\} \subseteq C_{\varrho}$ for some $\varrho>0$.

Proof of Theorem 3. Due to Lemma 2, the first part of (2.8), it suffices to to prove (3.1) for $i=1$. Moreover, thanks relation (3.1) of Lemma 3 we may assume that the function $g$ in (2.4) has bounded (non-empty) support, say $\operatorname{supp}(g) \subseteq C_{\varrho}$ for some $\varrho>0$. Hence, $g \in \boldsymbol{L}_{\mathbf{2}}\left(\mathbb{R}^{d}\right)$ implies $g \in \boldsymbol{L}_{\mathbf{1}}\left(\mathbb{R}^{d}\right)$.

We need further notation: For $z \in \mathbb{Z}^{d}$ define

$$
X_{z}^{(n)}:=\sum_{i \geq 1} \mathbf{1}_{E_{z} \cap W_{n}}\left(X_{i}\right) \sum_{j \neq i} g\left(X_{j}-X_{i}\right)-\lambda^{2}\left|E_{z} \cap W_{n}\right| J(g) \quad \text { with } \quad E_{z}=z+[-1 / 2,1 / 2)^{d} .
$$

By means of $V_{n}:=\left\{z \in \mathbb{Z}^{d}: E_{z} \subseteq W_{n}\right\}$ and $\partial V_{n}:=\left\{z \in \mathbb{Z}^{d}: E_{z} \cap W_{n}^{c} \neq \emptyset\right\}$ we rewrite $Y_{n, 1}(g)$ as partial sum process

$$
\sqrt{\left|W_{n}\right|} Y_{n, 1}(g)=\frac{1}{\left|W_{n}\right|} \sum_{z \in V_{n}} X_{z}^{(n)}+\frac{1}{\left|W_{n}\right|} \sum_{z \in \partial V_{n}} X_{z}^{(n)}
$$

over the $m$-dependent field of means zero random variables $\left\{X_{z}^{(n)}: z \in V_{n} \cup \partial V_{n}\right\}$ with $m=2\lceil\varrho\rceil+1$. For more information on (CLT's for) $m$-dependent fields the reader is referred e.g. to [15], [16], [18] and [7]. Note that the $m$-dependence results from the independence 
properties of the Poisson point process and that $g$ disappears outside $C_{\varrho}$. It is not difficult to see that

$$
\mathbf{E}\left(\sum_{z \in \partial V_{n}} X_{z}^{(n)}\right)^{2} \leq(m+1)^{d} \sum_{z \in \partial V_{n}} \mathbf{E}\left(X_{z}^{(n)}\right)^{2} \quad \text { with } \quad \mathbf{E}\left(X_{z}^{(n)}\right)^{2} \leq 2 \lambda^{2} J\left(g^{2}\right)+4 \lambda^{3} J(|g|)^{2} .
$$

The last estimate is obtained from a relation stated at the beginning of the proof of Lemma 3 . We next show that $\#\left(\partial V_{n}\right) \leq c_{3}(d)\left|\partial W_{n}\right|_{d-1}$. Obviously, $E_{z} \subseteq \partial W_{n} \oplus B(\mathbf{o}, \sqrt{d})$ for any $z \in \partial V_{n}$ implying \# $\left(\partial V_{n}\right) \leq\left|\partial W_{n} \oplus B(\mathbf{o}, \sqrt{d})\right|$. Due to the convexity of $W_{n}$ and by combining the first and second inequality of (1.2) we obtain that

$$
\left|\partial W_{n} \oplus B(\mathbf{o}, \sqrt{d})\right| \leq 2\left(\left|W_{n} \oplus B(\mathbf{o}, \varrho)\right|-\left|W_{n}\right|\right) \leq 2^{d} \sqrt{d}\left|\partial W_{n}\right|_{d-1},
$$

which yields the desired estimate with $c_{3}(d)=2^{d} \sqrt{d}$. By appealing to the properties of the CAS $\left(W_{n}\right)$ we get $\# V_{n} /\left|W_{n}\right| \underset{n \rightarrow \infty}{\longrightarrow} 1$ and the variance of the scaled second sum in (3.2) disappears asymptotically. Finally, we are in a position to apply the CLT for stationary mdependent fields (see e.g. [15] and references therein or the stronger result quoted in Remark 3) which provides that

$$
\left|W_{n}\right|^{-1 / 2} \sum_{z \in V_{n}} X_{z}^{(n)} \underset{n \rightarrow \infty}{\stackrel{\mathbf{d}}{\longrightarrow}} \mathcal{N}\left(0, \sigma^{2}(g)\right) .
$$

Together with Slutsky's theorem the latter implies (3.1) and completes the proof of Theorem 3.

Remark 5. By applying a Berry-Esseen bound proved in [7] for (non-stationary) m-dependent random fields in terms of the third-order Lyapunov ratio we can state the following: For any $g \mid \mathbb{R}^{d} \mapsto \mathbb{R}^{1}$ satisfying $\emptyset \neq \operatorname{supp}(g) \subseteq C_{\varrho}$ for some $\varrho>0$ and $g \in \boldsymbol{L}_{\mathbf{3}}\left(\mathbb{R}^{d}\right)$,

$$
\sup _{x \in \mathbb{R}^{1}}\left|\mathbf{P}\left(\sqrt{\left|W_{n}\right|} Y_{n, i}(g) \leq x \sigma(g)\right)-\mathbf{P}(\mathcal{N}(0,1) \leq x)\right| \leq \frac{c_{4}(d, \varrho, \lambda) J\left(|g|^{3}\right)}{\sigma^{3}(g) \sqrt{\left|W_{n}\right|}} \quad \text { for } \quad i=1,2,3 .
$$

Corollary 2. The FIDI's of the set-indexed processes $\left\{\sqrt{\left|W_{n}\right|} Y_{n, i}(B): B \in \mathcal{B}^{d} \cap C_{\varrho}\right\}$ defined in (1.8) for $i=1,2,3$ converge in distribution (as $n \rightarrow \infty$ ) to the FIDI's of the mean zero set-indexed Gaussian process $\left\{Y(B): B \in \mathcal{B}^{d} \cap C_{\varrho}\right\}$ with covariance function $C_{Y}(A, B):=$ $\lambda^{2}|A \cap B|+\lambda^{2}|A \cap(-B)|+4 \lambda^{3}|A||B|$.

Proof of Corollary 2. We put $g_{B_{1}, \ldots, B_{k}}^{\left(a_{1}, \ldots, a_{k}\right)}(x)=a_{1} \mathbf{1}_{B_{1}}(x)+\cdots+a_{k} \mathbf{1}_{B_{k}}(x)$ for any $a_{1}, \ldots, a_{k} \in \mathbb{R}^{1}$ (such that $\vee_{i=1}^{k}\left|a_{i}\right|>0$ ) and any $B_{1}, \ldots, B_{k} \in \mathcal{B}^{d} \cap C_{\varrho}$. Since $g_{B_{1}, \ldots, B_{k}}^{\left(a_{1}, \ldots, a_{k}\right)}$ fulfils the assumptions of Theorem 3 we deduce from (3.1) that

$$
\sqrt{\left|W_{n}\right|} Y_{n, i}\left(g_{B_{1}, \ldots, B_{k}}^{\left(a_{1}, \ldots, a_{k}\right)}\right) \underset{n \rightarrow \infty}{\stackrel{\mathbf{d}}{\longrightarrow}} \mathcal{N}\left(0, \sum_{p, q=1}^{k} a_{p} a_{q} C_{Y}\left(B_{p}, B_{q}\right)\right)
$$


Hence, by applying the classical Cramér-Wold device, see e.g. [4], it follows the convergence in distribution of the $k$-dimensional random vectors

$$
\left(\sqrt{\left|W_{n}\right|} Y_{n, i}\left(B_{1}\right), \ldots, \sqrt{\left|W_{n}\right|} Y_{n, i}\left(B_{k}\right)\right) \underset{n \rightarrow \infty}{\stackrel{\mathrm{d}}{\longrightarrow}}\left(Y\left(B_{1}\right), \ldots, Y\left(B_{k}\right)\right)
$$

for any $B_{1}, \ldots, B_{k} \in \mathcal{B}^{d} \cap C_{\varrho}$. Hence, Corollary 2 is proved.

Proof of Theorem 1. Corollary 2 implies immediately to weak convergence of the FIDI's of the multiparameter processes $\left\{Y_{n, i}(\mathbf{r})=\sqrt{\left|W_{n}\right|} Y_{n, i}(B(\mathbf{r})): \mathbf{r} \in I_{\varrho}\right\}$ to the FIDI's of the multiparameter Gaussian process $\left\{Y(\mathbf{r})=Y(B(\mathbf{r})): \mathbf{r} \in I_{\varrho}\right\}$. Since $B(\mathbf{r})=-B(\mathbf{r})$ and $|B(\mathbf{r})|=2^{d}|r|$ for $\mathbf{r} \in I_{\varrho}$ we get the covariance function $\mathbf{E} Y(\mathbf{s}) Y(\mathbf{t})=C_{Y}(B(\mathbf{s}), B(\mathbf{t}))=2 \lambda^{2}|B(\mathbf{s} \wedge \mathbf{t})|+$ $4 \lambda^{3}|B(\mathbf{s})||B(\mathbf{t})|=\lambda^{2} 2^{d+1}|\mathbf{s} \wedge \mathbf{t}|+\lambda^{3} 4^{d+1}|\mathbf{s}||\mathbf{t}|$ which coincides with the covariance function given in Theorem 1. A straightforward calculation shows that the covariance function of the Gaussian process $\left\{\lambda 2^{(d+1) / 2}\left(W(\mathbf{r})+\left(\sqrt{1+2^{d+1} \lambda \varrho^{d}}-1\right) \varrho^{-d}|\mathbf{r}| W(\varrho)\right): \mathbf{r} \in I_{\varrho}\right\}$ coincides with that of $\left\{Y(\mathbf{r}): \mathbf{r} \in I_{\varrho}\right\}$. The tightness of $\left\{Y_{n, i}(\mathbf{r}): \mathbf{r} \in I_{\varrho}\right\}$ stated in Lemma 4 completes the proof of Theorem 1 .

\section{CLT for the Sequences $\sqrt{\left|W_{n}\right|} Z_{n, i}(g)$ and Proof of Theorem 2}

For the sake of simplicity we prefer to treat the case $i=3$ with $Z_{n, 3}(g)$ defined in (2.7) which means to prove that

$$
\begin{aligned}
& \sqrt{\left|W_{n}\right|} Z_{n, 3}(g)=\frac{1}{\sqrt{\left|W_{n}\right|}}\left(\sum_{i, j \geq 1}^{\neq} \mathbf{1}_{W_{n}}\left(X_{i}\right) \mathbf{1}_{W_{n}}\left(X_{j}\right) g\left(X_{j}-X_{i}\right)-\left|W_{n}\right| \widehat{\left(\lambda^{2}\right)_{n}} J_{n}(g)\right) \\
& =\frac{1}{\sqrt{\left|W_{n}\right|}} \sum_{i, j \geq 1}^{\neq \neq} \mathbf{1}_{W_{n}}\left(X_{i}\right) \mathbf{1}_{W_{n}}\left(X_{j}\right)\left(g\left(X_{j}-X_{i}\right)-\frac{J_{n}(g)}{\left|W_{n}\right|}\right) \underset{n \rightarrow \infty}{\mathbf{d}} \mathcal{N}\left(0, \tau^{2}(g)\right)
\end{aligned}
$$

where $J_{n}(g)=\left|W_{n}\right|^{-1} \int g(x)\left|W_{n} \cap\left(W_{n}-x\right)\right| \mathrm{d} x$ and $\tau^{2}(g)=\lambda^{2} \int g(x)(g(x)+g(-x)) \mathrm{d} x$.

Whereas the proof of Theorem 3 relies on the CLT for $m$-dependent random fields, we will prove (4.1) by conditioning on $\left\{N_{\lambda}\left(W_{n}\right)=m_{n}\right\}$ such that $m_{n} /\left|W_{n}\right| \underset{n \rightarrow \infty}{\longrightarrow} \lambda>0$ and showing asymptotic normality of a $U$-statistic defined for a triangular array of independent, uniformly distributed (short: I.U.D.) random points on $W_{n}$.

Lemma 5. Let $X_{1}^{(n)}, \ldots, X_{m_{n}}^{(n)}$ be a triangular array of I.U.D. random vectors on $W_{n}$ such that $m_{n} /\left|W_{n}\right| \underset{n \rightarrow \infty}{\longrightarrow} \lambda>0$. Then, for any $g \in \boldsymbol{L}_{\mathbf{1}}\left(\mathbb{R}^{d}\right) \cap \boldsymbol{L}_{\mathbf{2}}\left(\mathbb{R}^{d}\right)$,

$$
U_{n}(g):=\frac{1}{\sqrt{\left|W_{n}\right|}} \sum_{1 \leq i, j \leq m_{n}}^{\neq}\left(g\left(X_{i}^{(n)}-X_{j}^{(n)}\right)-\frac{J_{n}(g)}{\left|W_{n}\right|}\right) \underset{n \rightarrow \infty}{\stackrel{\mathbf{d}}{\longrightarrow}} \mathcal{N}\left(0, \tau^{2}(g)\right)
$$

with $J_{n}(g)$ and $\tau^{2}(g)$ as defined in (4.1). 
Proof of Lemma 5. It turns out that the Hoeffdings "projection method", see Chapt. 5.3 in [38], fails due to the fact that the $U$-statistic $\sqrt{\left|W_{n}\right|} U_{n}(g)$ is asymptotically degenerate. Some similar spatial $U$-statistics with Gaussian limits in [24] do not include our specific situation. Lemma 5 extends a CLT for interpoint-distances in [14] and is based on Bolthausen's lemma (which is a variant of the Stein-Tikhomirov method), see [5]. In accordance with this lemma, (4.2) holds if we can prove with the characteristic function $f_{n}(t):=\mathbf{E} \exp \left\{i t U_{n}(g)\right\}$ that

$$
\mathbf{E} U_{n}^{2}(g) \underset{n \rightarrow \infty}{\longrightarrow} \tau^{2}(g) \quad \text { and } \quad f_{n}^{\prime}(t)+\tau^{2}(g) t f_{n}(t) \underset{n \rightarrow \infty}{\longrightarrow} 0 \text { for each } t \in \mathbb{R}^{1}
$$

First we calculate $\mathbf{E} U_{n}^{2}(g)$ in analogy to (2.15). With the abbreviations of Sect. 2 we arrive at

$$
\begin{aligned}
\mathbf{E} U_{n}^{2}(g) & =\frac{m_{n}\left(m_{n}-1\right)}{\left|W_{n}\right|^{2}} \int g(x)(g(x)+g(-x)) R_{n}(x) \mathrm{d} x-\frac{m_{n}\left(m_{n}-1\right)\left(4 m_{n}-6\right)}{\left|W_{n}\right|^{3}}\left(J_{n}(g)\right)^{2} \\
& +\frac{m_{n}\left(m_{n}-1\right)\left(m_{n}-2\right)}{\left|W_{n}\right|^{3}} \iint(g(x)+g(-x))(g(y)+g(-y)) R_{n}(x, y) \mathrm{d} x \mathrm{~d} y .
\end{aligned}
$$

Lebesgue's dominated convergence theorem and $m_{n} /\left|W_{n}\right| \underset{n \rightarrow \infty}{\longrightarrow} \lambda$ yield $\mathbf{E} U_{n}^{2}(g) \underset{n \rightarrow \infty}{\longrightarrow} \tau_{n}^{2}(g)$. With the truncated function $g_{a, \varrho}(x)=g(x)$, if $x \in C_{\varrho}$ and $|g(x)| \leq a$, and $g(x)=0$ otherwise, for $a, \varrho>0$, and the above formula for $\mathbf{E} U_{n}^{2}(g)$ permits the estimate

$$
\mathbf{E}\left(U_{n}(g)-U_{n}\left(g_{a, \varrho}\right)\right)^{2} \leq 2 \lambda_{n}^{2} c_{2}(a, \varrho)+8 \lambda_{n}^{3} c_{1}(a, \varrho)^{2},
$$

where $\lambda_{n}=m_{n} /\left|W_{n}\right|$ and $c_{1}(a, \varrho), c_{2}(a, \varrho)$ as in Lemma 3. The latter estimate combined with Slutsky's theorem, see [38], enables us in proving the second limit of (4.3) to assume additionally that $|g(x)| \leq a$ for $x \in \operatorname{supp}(g) \subseteq C_{\varrho}$ for some finite $a, \varrho>0$. Next, we will write $\int_{\left(x_{i}\right)}$ shorthand for an integral over $W_{n}$ w.r.t. the variable $x_{i}$. With the abbreviation

$$
H_{n}(z):=g(z)+g(-z)-2 J_{n}(g) /\left|W_{n}\right| \text { and } \int_{(x)} \int_{(y)} H_{n}(x-y) \mathrm{d} x \mathrm{~d} y=0
$$

we may write

$$
f_{n}(t)=\int_{\left(x_{1}\right)} \cdots \int_{\left(x_{m_{n}}\right)} \exp \left\{\frac{i t}{\sqrt{\left|W_{n}\right|}} \sum_{1 \leq i<j \leq m_{n}}^{\neq} H_{n}\left(x_{i}-x_{j}\right)\right\} \frac{\mathrm{d} x_{1}}{\left|W_{n}\right|} \cdots \frac{\mathrm{d} x_{m_{n}}}{\left|W_{n}\right|}
$$


and get the decomposition

$$
\begin{aligned}
& f_{n}^{\prime}(t)=i \frac{m_{n}\left(m_{n}-1\right)}{2 \sqrt{\left|W_{n}\right|}} \int_{\left(x_{1}\right)} \int_{\left(x_{2}\right)} H_{n}\left(x_{1}-x_{2}\right) \int_{\left(x_{3}\right)} \cdots \int_{\left(x_{m_{n}}\right)} \exp \left\{\frac { i t } { \sqrt { | W _ { n } | } } \left[H_{n}\left(x_{1}-x_{2}\right)\right.\right. \\
& \left.\left.+\sum_{i=3}^{m_{n}}\left(H_{n}\left(x_{1}-x_{i}\right)+H_{n}\left(x_{2}-x_{i}\right)\right)+\sum_{3 \leq i<j \leq m_{n}}^{\neq} H_{n}\left(x_{i}-x_{j}\right)\right]\right\} \frac{\mathrm{d} x_{1}}{\left|W_{n}\right|} \cdots \frac{\mathrm{d} x_{m_{n}}}{\left|W_{n}\right|} \\
& =i \frac{m_{n}\left(m_{n}-1\right)}{2 \sqrt{\left|W_{n}\right|}} \int_{\left(x_{1}\right)} \int_{\left(x_{2}\right)} H_{n}\left(x_{1}-x_{2}\right) \int_{\left(x_{3}\right)} \cdots \int_{\left(x_{m_{n}}\right)}\left(\operatorname { e x p } \left\{\frac { i t } { \sqrt { | W _ { n } | } } \left[H_{n}\left(x_{1}-x_{2}\right)\right.\right.\right. \\
& \left.\left.+\sum_{i=3}^{m_{n}}\left(H_{n}\left(x_{1}-x_{i}\right)+H_{n}\left(x_{2}-x_{i}\right)\right)\right]\right\}-1-\frac{i t}{\sqrt{\left|W_{n}\right|}}\left[H_{n}\left(x_{1}-x_{2}\right)\right. \\
& \left.\left.+\sum_{i=3}^{m_{n}}\left(H_{n}\left(x_{1}-x_{i}\right)+H_{n}\left(x_{2}-x_{i}\right)\right)\right]\right) \exp \left\{\frac{i t}{\sqrt{\left|W_{n}\right|}} \sum_{3 \leq i<j \leq m_{n}}^{\neq} H_{n}\left(x_{i}-x_{j}\right)\right\} \frac{\mathrm{d} x_{1}}{\left|W_{n}\right|} \cdots \frac{\mathrm{d} x_{m_{n}}}{\left|W_{n}\right|} \\
& -\frac{m_{n}\left(m_{n}-1\right) t}{2\left|W_{n}\right|} \int_{\left(x_{1}\right)} \int_{\left(x_{2}\right)} H_{n}^{2}\left(x_{1}-x_{2}\right) \frac{\mathrm{d} x_{1}}{\left|W_{n}\right|} \frac{\mathrm{d} x_{2}}{\left|W_{n}\right|} f_{n}^{\diamond}(t) \\
& -\frac{m_{n}\left(m_{n}-1\right)\left(m_{n}-2\right) t}{\left|W_{n}\right|} \int_{\left(x_{1}\right)} \ldots \int_{\left(x_{m_{n}}\right)} H_{n}\left(x_{1}-x_{2}\right) H_{n}\left(x_{1}-x_{3}\right) \\
& \times \exp \left\{\frac{i t}{\sqrt{\left|A_{n}\right|}} \sum_{3 \leq i<j \leq m_{n}}^{\neq} H_{n}\left(x_{i}-x_{j}\right)\right\} \frac{\mathrm{d} x_{1}}{\left|W_{n}\right|} \cdots \frac{\mathrm{d} x_{m_{n}}}{\left|W_{n}\right|} \\
& =T_{1}^{(n)}(t)+T_{2}^{(n)}(t)+T_{3}^{(n)}(t),
\end{aligned}
$$

where

$$
f_{n}^{\diamond}(t):=\int_{\left(x_{3}\right)} \cdots \int_{\left(x_{m_{n}}\right)} \exp \left\{\frac{i t}{\sqrt{\left|W_{n}\right|}} \sum_{3 \leq i<j \leq m_{n}}^{\neq} H_{n}\left(x_{i}-x_{j}\right)\right\} \frac{\mathrm{d} x_{3}}{\left|W_{n}\right|} \cdots \frac{\mathrm{d} x_{m_{n}}}{\left|W_{n}\right|}
$$

Due to the well-known inequality $\left|e^{i t x}-1-i t x\right| \leq t^{2} x^{2} / 2$ for any $t, x \in \mathbb{R}^{1}$ we obtain that

$$
\begin{aligned}
\left|T_{1}^{(n)}(t)\right| & \leq \frac{m_{n}\left(m_{n}-1\right) t^{2}}{4 \sqrt{\left|W_{n}\right|}\left|W_{n}\right|} \int_{\left(x_{1}\right)} \cdots \int_{\left(x_{m_{n}}\right)}\left|H_{n}\left(x_{1}-x_{2}\right)\right| \\
& \times\left(H_{n}\left(x_{1}-x_{2}\right)+\sum_{i=3}^{m_{n}}\left(H_{n}\left(x_{1}-x_{i}\right)+H_{n}\left(x_{2}-x_{i}\right)\right)\right)^{2} \frac{\mathrm{d} x_{1}}{\left|W_{n}\right|} \cdots \frac{\mathrm{d} x_{m_{n}}}{\left|W_{n}\right|} \\
& \leq \frac{m_{n}\left(m_{n}-1\right) t^{2}}{4 \sqrt{\left|W_{n}\right|}\left|W_{n}\right|^{3}}\left(I_{1}^{(n)}+\frac{8\left(m_{n}-2\right)}{\left|W_{n}\right|} I_{2}^{(n)}+\frac{4\left(m_{n}-2\right)\left(m_{n}-3\right)}{\left|W_{n}\right|^{2}} I_{3}^{(n)}\right)
\end{aligned}
$$


with

$$
\begin{aligned}
I_{1}^{(n)} & =\int_{\left(x_{1}\right)} \int_{\left(x_{2}\right)}\left|H_{n}\left(x_{1}-x_{2}\right)\right|^{3} \mathrm{~d} x_{1} \mathrm{~d} x_{2} \leq 8 c \sigma^{2}(g)\left|W_{n}\right| \\
I_{2}^{(n)} & =\int_{\left(x_{1}\right)} \int_{\left(x_{2}\right)} \int_{\left(x_{3}\right)} H_{n}^{2}\left(x_{1}-x_{2}\right)\left|H_{n}\left(x_{1}-x_{3}\right)\right| \mathrm{d} x_{1} \mathrm{~d} x_{2} \mathrm{~d} x_{3} \leq 8 \int|g(x)| \mathrm{d} x \sigma^{2}(g)\left|W_{n}\right| \\
I_{3}^{(n)} & =\int_{\left(x_{1}\right)} \cdots \int_{\left(x_{4}\right)}\left|H_{n}\left(x_{1}-x_{2}\right)\right|\left|H_{n}\left(x_{1}-x_{3}\right)\right|\left(\left|H_{n}\left(x_{1}-x_{4}\right)\right|+\left|H_{n}\left(x_{2}-x_{4}\right)\right|\right) \mathrm{d} x_{1} \ldots \mathrm{d} x_{4} \\
& \leq 2\left(4 \int|g(x)| \mathrm{d} x\right)^{3}\left|W_{n}\right| .
\end{aligned}
$$

The above estimates follow immediately from $|H(x)| \leq 4 a$ (since $|g(x)| \leq a)$, $\int_{(y)}\left|H_{n}(x-y)\right| \mathrm{d} y \leq 4 \int|g(y)| \mathrm{d} y$ for all $x \in \mathbb{R}^{1}$ and the relation

$\int_{\left(x_{1}\right)} \int_{\left(x_{2}\right)} H_{n}^{2}\left(x_{1}-x_{2}\right) \mathrm{d} x_{1} \mathrm{~d} x_{2}=\left|W_{n}\right| \int(g(x)+g(-x))^{2} R_{n}(x) \mathrm{d} x-4 J_{n}^{2}(g) \leq 2 \tau^{2}(g)\left|W_{n}\right|$, which implies that

$$
\left|W_{n}\right|^{-1} \int_{\left(x_{1}\right)} \int_{\left(x_{2}\right)} H_{n}^{2}\left(x_{1}-x_{2}\right) \mathrm{d} x_{1} \mathrm{~d} x_{2} \underset{n \rightarrow \infty}{\longrightarrow} 2 \tau^{2}(g)
$$

Summing up all terms on the r.h.s. of (4.5) yields

$$
T_{1}^{(n)}(t)=t^{2} \mathcal{O}\left(\left|W_{n}\right|^{-1 / 2}\right) \quad \text { as } \quad n \rightarrow \infty .
$$

From the definition of $T_{3}^{(n)}(t)$ it is rapidly seen that

$$
\left|T_{3}^{(n)}(t)\right| \leq \frac{m_{n}\left(m_{n}-1\right)\left(m_{n}-2\right)|t|}{\left|W_{n}\right|^{4}} \int_{\left(x_{1}\right)}\left|\int_{\left(x_{2}\right)} H_{n}\left(x_{1}-x_{2}\right) \mathrm{d} x_{2}\right| \mathrm{d} x_{1} \sup _{x_{1} \in W_{n}} \int_{\left(x_{3}\right)}\left|H_{n}\left(x_{1}-x_{3}\right)\right| \mathrm{d} x_{3},
$$

where last integral over $x_{3}$ is bounded by $4 \int|g(x)| \mathrm{d} x$. To find an appropriate bound of the remaining integral term we remember the fact that the support of $g$ is contained in the cube $C_{\varrho}$ centred at the origin o. Using the abbreviation $W_{n}^{(\varrho)}:=\left\{x \in W_{n}: B(x, \varrho \sqrt{d}) \subseteq W_{n}\right\}$, it follows that $\int_{(y)} g(y-x) \mathrm{d} y=\int g(y) \mathrm{d} y$ for any $x \in W_{n}^{(\varrho)}$ and

$$
\left|\int g(x)\right| W_{n} \cap\left(W_{n}-x\right)|\mathrm{d} x-| W_{n}^{(\varrho)}\left|\int g(x) \mathrm{d} x\right| \leq\left|W_{n} \backslash W_{n}^{(\varrho)}\right| \int|g(x)| \mathrm{d} x .
$$

Having in mind these relations and splitting the domain of integration over $x_{1}$ into $W_{n}^{(\varrho)}$ and $W_{n} \backslash W_{n}^{(\varrho)}$ we arrive after some rearrangements at 


$$
\int_{W_{n}}\left|\int_{W_{n}} H_{n}\left(x_{1}-x_{2}\right) \mathrm{d} x_{2}\right| \mathrm{d} x_{1} \leq 8\left|W_{n} \backslash W_{n}^{(\varrho)}\right| \int|g(x)| \mathrm{d} x
$$

Thus, $T_{3}^{(n)}(t)=|t| \mathcal{O}\left(\left|W_{n} \backslash W_{n}^{(\varrho)}\right| /\left|W_{n}\right|\right)$ as $n \rightarrow \infty$. This result combined with the properties of the CAS $\left(W_{n}\right)$ and (4.7) leads to

$$
f_{n}^{\prime}(t)+\frac{m_{n}\left(m_{n}-1\right) t}{2\left|W_{n}\right|} \int_{\left(x_{1}\right)} \int_{\left(x_{2}\right)} H_{n}^{2}\left(x_{1}-x_{2}\right) \frac{\mathrm{d} x_{1}}{\left|W_{n}\right|} \frac{\mathrm{d} x_{2}}{\left|W_{n}\right|} f_{n}^{\diamond}(t) \underset{n \rightarrow \infty}{\longrightarrow} 0
$$

for any fixed $t \in \mathbb{R}^{1}$. Further, by the inequality $\left|e^{i t x}-1\right| \leq|t x|$ for $t, x \in \mathbb{R}^{1}$, it follows that

$$
\begin{aligned}
\left|f_{n}^{\diamond}(t)-f_{n}(t)\right| & \leq \frac{\left(2 m_{n}-3\right)|t|}{\sqrt{W_{n}}\left|W_{n}\right|^{2}} \int_{\left(x_{1}\right)} \int_{\left(x_{2}\right)}\left|H_{n}\left(x_{1}-x_{2}\right)\right| \mathrm{d} x_{1} \mathrm{~d} x_{2} \\
& \leq \frac{4\left(2 m_{n}-3\right)|t|}{\sqrt{W_{n}}\left|W_{n}\right|} \int|g(x)| \mathrm{d} x \underset{n \rightarrow \infty}{\longrightarrow} 0 .
\end{aligned}
$$

Finally, together with the foregoing relation and (4.6), the second limit of (4.3) is proved completing the proof of Lemma 5.

Theorem 4. For any $g \in \boldsymbol{L}_{\mathbf{1}}\left(\mathbb{R}^{d}\right) \cap \boldsymbol{L}_{\mathbf{2}}\left(\mathbb{R}^{d}\right)$ and $i=1$ or $i=3$,

$$
\sqrt{\left|W_{n}\right|} Z_{n, i}(g) \underset{n \rightarrow \infty}{\stackrel{\mathbf{d}}{\longrightarrow}} \mathcal{N}\left(0, \tau^{2}(g)\right) \quad \text { with } \quad \tau^{2}(g)=\lambda^{2} \int g(x)(g(x)+g(-x)) \mathrm{d} x .
$$

Furthermore, (4.8) holds for $i=2$, if $g \in \boldsymbol{L}_{\mathbf{2}}\left(\mathbb{R}^{d}\right)$ and $\operatorname{supp}(g):=\{x: g(x) \neq 0\} \subseteq C_{\varrho}$ for some $\varrho>0$.

Proof of Theorem 4. By virtue of the second part of (2.8) we need only to verify (4.1). For this end, we make use of the total probability theorem providing that

$$
\mathbf{P}\left(\sqrt{\left|W_{n}\right|} Z_{n, 3}(g) \leq x\right)=\sum_{k \geq 0} \mathbf{P}\left(\sqrt{\left|W_{n}\right|} Z_{n, 3}(g) \leq x \mid N_{\lambda}\left(W_{n}\right)=k\right) \mathbf{P}\left(N_{\lambda}\left(W_{n}\right)=k\right)
$$

which immediately leads to

$$
\begin{gathered}
\left|\mathbf{P}\left(\sqrt{\left|W_{n}\right|} Z_{n, 3}(g) \leq x\right)-\mathbf{P}\left(\mathcal{N}\left(0, \tau^{2}(g)\right) \leq x\right)\right| \leq 1-\mathbf{P}\left(a_{n} \leq N_{\lambda}\left(W_{n}\right) \leq b_{n}\right) \\
+\mathbf{P}\left(a_{n} \leq N_{\lambda}\left(W_{n}\right) \leq b_{n}\right) \max _{a_{n} \leq m_{n} \leq b_{n}}\left|\mathbf{P}\left(\sqrt{\left|W_{n}\right|} Z_{n, 3}(g) \leq x \mid N_{\lambda}\left(W_{n}\right)=m_{n}\right)-\mathbf{P}\left(\mathcal{N}\left(0, \tau^{2}(g)\right) \leq x\right)\right|
\end{gathered}
$$

with the sequences of integers $a_{n}:=\left\lceil\lambda\left|W_{n}\right|-\lambda_{n}\left|W_{n}\right|^{1 / 2}\right\rceil$ and $b_{n}:=\left\lceil\lambda\left|W_{n}\right|+\lambda_{n}\left|W_{n}\right|^{1 / 2}\right\rceil$, where $\lambda_{n}=o\left(\left|W_{n}\right|^{1 / 2}\right)$. Since $\mathbf{P}\left(\sqrt{\left|W_{n}\right|} Z_{n, 3}(g) \leq x \mid N_{\lambda}\left(W_{n}\right)=m_{n}\right)=\mathbf{P}\left(U_{n}(g) \leq x\right)$ due to the CSR-property of $N_{\lambda}$, Lemma 5 and $\hat{\lambda}_{n} \underset{n \rightarrow \infty}{\stackrel{\mathbf{P}-a . s .}{\longrightarrow}} \lambda$ imply the validity of (4.1). 
Remark 6. So far the rates of convergence in the Theorem 4 as well as in Lemma 5 are unknown. It seems that the methods applied in the recent papers [36] and [30] can help to answer this open question.

Corollary 3. The FIDI's of the set-indexed processes $\left\{Z_{n, i}(B): B \in \mathcal{B}^{d} \cap C_{\varrho}\right\}$ defined in (1.9) for $i=1,2,3$ converge in distribution (as $n \rightarrow \infty$ ) to the FIDI's of the mean zero set-indexed Gaussian process $\left\{Z(B): B \in \mathcal{B}^{d} \cap C_{\varrho}\right\}$ possessing the covariance function $C_{Z}(A, B)=$ $\lambda^{2}|A \cap B|+\lambda^{2}|A \cap(-B)|$.

The proof of Corollary 3 consists in repeating almost verbatim the proof of Corollary 2 .

Proof of Theorem 2. Corollary 3 implies the weak convergence of the FIDI's of the multiparameter processes $\left\{Z_{n, i}(\mathbf{r})=\sqrt{\left|W_{n}\right|} Z_{n, i}(B(\mathbf{r})): \mathbf{r} \in I_{\varrho}\right\}$ to the FIDI's of the multiparameter Gaussian process $\left\{Z(\mathbf{r})=Z(B(\mathbf{r})): \mathbf{r} \in I_{\varrho}\right\}$ having the covariance function $C_{Z}(B(\mathbf{s}), B(\mathbf{t}))=2 \lambda^{2}|B(\mathbf{s} \wedge \mathbf{t})|=\lambda^{2} 2^{d+1}|\mathbf{s} \wedge \mathbf{t}|$. The latter shows that $\left\{Y(\mathbf{r}): \mathbf{r} \in I_{\varrho}\right\}$ and $\left\{\lambda 2^{(d+1) / 2} W(\mathbf{r}): \mathbf{r} \in I_{\varrho}\right\}$ have the same FIDI's. The tightness of $\left\{Z_{n, i}(\mathbf{r}): \mathbf{r} \in I_{\varrho}\right\}$ stated in Lemma 4 completes the proof of Theorem 2 .

\section{Applications to Testing for Complete Spatial Randomness}

From the viewpoint of spatial point process statistics the main reason for studying limits of set-indexed or multiparameter empirical processes consists in the construction of asymptotic goodness-of-fit tests to check non-parametric characteristics of the spatial point process model under consideration. Theorems 1 and 2 allow to establish Kolmogorov-Smirnov and Cramér-von Mises type tests, respectively, for the multiparameter $K$-function of a stationary Poisson process (multiplied by $\lambda^{2}$ ) for the cases of known as well as estimated intensity $\lambda$. These goodness-of-fit tests can be interpreted as test for CSR generalizing the tests based on the empirical one-parameter $K$-function suggested in [16]. For alternative (mostly nonasymptotic) tests for CSR the reader is referred to [12], [23], [13], [22], [42] and further references therein.

For this purpose, we are interested in the limit distributions of the test statistics

$$
K_{Y}^{(n)}(\lambda, d, \varrho)=\frac{1}{\sqrt{\lambda}} \max _{\mathbf{r} \in I_{\varrho}}\left|Y_{n, 2}(\mathbf{r})\right| \quad, \quad C_{Y}^{(n)}(\lambda, d, \varrho)=\frac{1}{\lambda} \int_{I_{\varrho}} Y_{n, 2}^{2}(\mathbf{r}) \mathrm{d} \mathbf{r}
$$

and

$$
K_{Z}^{(n)}(d, \varrho)=\frac{1}{\widehat{\lambda}_{n} \sqrt{2^{d+1} \varrho^{d}}} \max _{\mathbf{r} \in I_{\varrho}}\left|Z_{n, 2}(\mathbf{r})\right| \quad, \quad C_{Z}^{(n)}(d, \varrho)=\frac{1}{\hat{\lambda}_{n}^{2} 2^{d+1} \varrho^{d}} \int_{I_{\varrho}} Z_{n, 2}^{2}(\mathbf{r}) \mathrm{d} \mathbf{r} .
$$


Using the continuity of the mappings $f \mapsto \max _{\mathbf{r} \in I_{\varrho}}|f(\mathbf{r})|$ and $f \mapsto \int_{I_{\varrho}} f(\mathbf{r})^{2} \mathrm{~d} \mathbf{r}$ on the Skorokhod-space $\boldsymbol{D}[0, \varrho]^{d}$ we may combine the continuous mapping theorem, see [4], Slutsky's theorem, see [38], and the Theorems 1 and 2 to obtain

$$
\begin{aligned}
& K_{Y}^{(n)}(\lambda, d, \varrho) \underset{n \rightarrow \infty}{\stackrel{\mathbf{d}}{\longrightarrow}} \frac{1}{\sqrt{\lambda}} \max _{\mathbf{r} \in I_{\varrho}}|Y(\mathbf{r})| \\
& \stackrel{\mathbf{d}}{=} \quad \sqrt{\lambda 2^{d+1}} \max _{\mathbf{r} \in I \varrho}\left|W(\mathbf{r})+\left(\sqrt{1+2^{d+1} \lambda \varrho^{d}}-1\right) \varrho^{-d}\right| \mathbf{r}|W(\varrho)| \\
& \stackrel{\mathbf{d}}{=} \quad \sqrt{\kappa} \max _{\mathbf{r} \in I_{1}}|W(\mathbf{r})+(\sqrt{1+\kappa}-1)| \mathbf{r}|W(\boldsymbol{e})|=: \Delta_{d, \kappa}
\end{aligned}
$$

where $\boldsymbol{e}=(1, \ldots, 1), \kappa=\lambda 2^{d+1} \varrho^{d}$ and

$$
\begin{aligned}
& K_{Z}^{(n)}(d, \varrho) \underset{n \rightarrow \infty}{\stackrel{\mathbf{d}}{\longrightarrow}} \varrho^{-d / 2} \max _{\mathbf{r} \in I_{\varrho}}|W(\mathbf{r})| \stackrel{\mathbf{d}}{=} \max _{\mathbf{r} \in I_{1}}|W(\mathbf{r})|=: K_{d}, \\
& C_{Z}^{(n)}(d, \varrho) \underset{n \rightarrow \infty}{\stackrel{\mathbf{d}}{\longrightarrow}} \varrho^{-d} \int_{I_{\varrho}} W(\mathbf{r})^{2} \mathrm{~d} \mathbf{r} \stackrel{\mathbf{d}}{=} \int_{I_{1}} W^{2}(\mathbf{r}) \mathrm{d} \mathbf{r}=: C_{d} .
\end{aligned}
$$

Here we have used the scale invariance $\left\{\varrho^{-d / 2} W(\varrho \mathbf{r}): \mathbf{r} \in \mathbb{R}_{+}^{d}\right\} \stackrel{\mathbf{d}}{=}\left\{W(\mathbf{r}): \mathbf{r} \in \mathbb{R}_{+}^{d}\right\}$ of the Wiener sheet on $\mathbb{R}_{+}^{d}$, see [1], entailing that $\left\{\sqrt{\lambda 2^{d+1}}\left(W(\mathbf{r})+\left(\sqrt{1+2^{d+1} \lambda \varrho^{d}}-\right.\right.\right.$ 1) $\left.\left.\varrho^{-d}|\mathbf{r}| W(\varrho)\right): \mathbf{r} \in I_{\varrho}\right\} \stackrel{\mathbf{d}}{=}\left\{\sqrt{\kappa}(W(\mathbf{r})+(\sqrt{1+\kappa}-1)|\mathbf{r}| W(\boldsymbol{e})): \mathbf{r} \in I_{1}\right\}$. Note that explicit expressions neither for the distribution functions $H_{d, \kappa}, F_{d}$ and $G_{d}$ of $\Delta_{\kappa}, K_{d}$ and $C_{d}$, respectively, nor for their densities (they do exist!) are known. Only their asymptotic tail behaviour seems to be available, see [2] and references therein. It is well-known that the random variable $C_{d}$ can be represented as infinite quadratic form of I.I.D. $\mathcal{N}(0,1)$-distributed random variables, see e.g. [33], more precisely,

$$
C_{d} \stackrel{\mathbf{d}}{=}\left(\frac{2}{\pi}\right)^{2 d} \sum_{i_{1}, \ldots, i_{d}=1}^{\infty} \frac{\xi_{i_{1}, \ldots, i_{d}}^{2}}{\left(2 i_{1}-1\right)^{2} \cdots\left(2 i_{d}-1\right)^{2}} \quad \text { with I.I.D. } \quad \xi_{i_{1}, \ldots, i_{d}} \stackrel{\mathbf{d}}{=} \mathcal{N}(0,1)
$$

providing the characteristic function

$$
\mathbf{E} \exp \left\{i t C_{d}\right\}=\prod_{i_{1}, \ldots, i_{d}=1}^{\infty}\left(1-\frac{2^{2 d+1} i t}{\pi^{2 d}\left(2 i_{1}-1\right)^{2} \cdots\left(2 i_{d}-1\right)^{2}}\right)^{-1 / 2} \text { for } \quad t \in \mathbb{R}^{1}
$$

If $i t$ is replaced by $-a \leq 0$ we get a formula for the Laplace-Stieltjes transform of $G_{d}$. In [11] using different methods from stochastic analysis the shape of the Laplace-Stieltjes transform of $G_{2}$ was shown to be

$$
\mathbf{E} \exp \left\{-a C_{2}\right\}=\left(\prod_{k=1}^{\infty} \cosh \left(\frac{2 \sqrt{2 a}}{(2 k-1) \pi}\right)\right)^{-1 / 2} \quad \text { for } \quad a \geq 0
$$

By combining analytical techniques and numerical procedures it might be possible to invert $\mathbf{E} \exp \left\{-a C_{2}\right\}$ at least approximately, see [32],[29] and [33] for the principle ideas and an 
application to the bivariate Brownian bridge. In our situation it remains to determine best possible approximations for the $\mathbf{1 0 0} \boldsymbol{\alpha} \%$ percentage points $H_{d, \kappa}(\alpha)=\inf \left\{x>0: H_{d, \kappa}(x) \geq\right.$ $\alpha\}, F_{d}(\alpha)=\inf \left\{x>0: F_{d}(x) \geq \alpha\right\}$ and $G_{d}(\alpha)=\inf \left\{x>0: G_{d}(x) \geq \alpha\right\}$ by a large-scale simulation of the Wiener sheet on $[0,1]^{d}$ and an estimation of the corresponding densities of $H_{d, \kappa}, F_{d}$ and $G_{d}$. For $d=2$ we have simulated 100,000 realizations of the Wiener sheet on an equidistant grid of $1,000 \times 1,000$ points in $[0,1]^{2}$. The obtained percentage points are summarized in the subsequent tables.

Table of $100 \alpha \%$ percentage points of $F_{2}$ and $G_{2}$

\begin{tabular}{|c|c|c|c|c|c|c|c|c|c|c|}
\hline $100 \alpha \%$ & $95 \%$ & $95.5 \%$ & $96 \%$ & $96.5 \%$ & $97 \%$ & $97.5 \%$ & $98 \%$ & $98.5 \%$ & $99 \%$ & $99.5 \%$ \\
\hline$F_{2}(\alpha]$ & 2.434 & 2.476 & 2.514 & 2.555 & 2.671 & 2.671 & 2.747 & 2.839 & 2.971 & 3.180 \\
\hline$G_{2}(\alpha)$ & 0.713 & 0.745 & 0.778 & 0.814 & 0.856 & 0.909 & 0.974 & 1.056 & 1.170 & 1.387 \\
\hline
\end{tabular}

Table of $100 \alpha \%$ percentage points of $H_{2, \kappa}$ for $\kappa=0.5+0.1 \times k, k=0,1, \ldots, 25$

\begin{tabular}{|c|c|c|c|c|c|c|c|c|c|c|}
\hline$\kappa$ & $95 \%$ & $97.5 \%$ & $99 \%$ & $99.5 \%$ & & $\kappa$ & $95 \%$ & $97.5 \%$ & $99 \%$ & $99.5 \%$ \\
\hline 0.5 & 1.966 & 2.179 & 2.440 & 2.636 & & 1.8 & 4.765 & 5.336 & 6.038 & 6.556 \\
0.6 & 2.206 & 2.447 & 2.741 & 2.965 & & 1.9 & 4.969 & 5.565 & 6.301 & 6.843 \\
0.7 & 2.437 & 2.708 & 3.036 & 3.285 & & 2.0 & 5.170 & 5.794 & 6.561 & 7,129 \\
0.8 & 2.662 & 2.961 & 3.324 & 3.601 & & 2.1 & 5.372 & 6.020 & 6.822 & 7.414 \\
0.9 & 2.882 & 3.209 & 3.610 & 3.904 & & 2.2 & 5.573 & 6.246 & 7.085 & 7.696 \\
1.0 & 3.099 & 3.451 & 3.887 & 4.207 & & 2.3 & 5.775 & 6.474 & 7.345 & 7.978 \\
1.1 & 3.314 & 3.692 & 4.160 & 4.509 & & 2.4 & 5.977 & 6.701 & 7.607 & 8.263 \\
1.2 & 3.527 & 3.934 & 4.432 & 4.809 & & 2.5 & 6.179 & 6.932 & 7.867 & 8.543 \\
1.3 & 3.736 & 4.171 & 4.701 & 5.111 & & 2.6 & 6.379 & 7.161 & 8.124 & 8.826 \\
1.4 & 3.944 & 4.405 & 4.972 & 5.403 & & 2.7 & 6.579 & 7.390 & 8.387 & 9.109 \\
1.5 & 4.150 & 4.637 & 5.243 & 5.690 & & 2.8 & 6.781 & 7.614 & 8.646 & 9.391 \\
1.6 & 4.356 & 4.873 & 5.509 & 5.983 & & 2.9 & 6.978 & 7.839 & 8.902 & 9.676 \\
1.7 & 4.563 & 5.105 & 5.774 & 6.269 & & 3.0 & 7.179 & 8.067 & 9.167 & 9.957 \\
\hline
\end{tabular}

Acknowledgements. The author is deeply indebted to Dr. F. Reffel for his support in the computation of the above-given $100 \alpha \%$ percentage points based on large-scale simulations of the two-dimensional Wiener sheet and the distribution functions $F_{2}, G_{2}$ and $H_{2, \kappa}$. 
The computation of the subsequent plots presenting the distribution function and probability density of $K_{2}$ and $C_{2}$, respectively, are based on 100,000 simulations of the standard Wiener sheet on an equidistant $1,000 \times 1,000$ grid in $[0,1]^{2}$

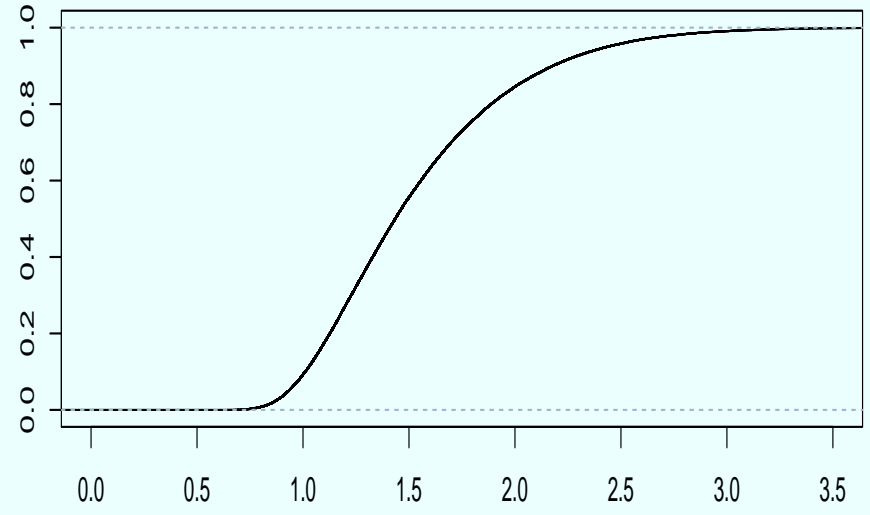

Fig.4: Empirical distribution function of $F_{2}(x)$

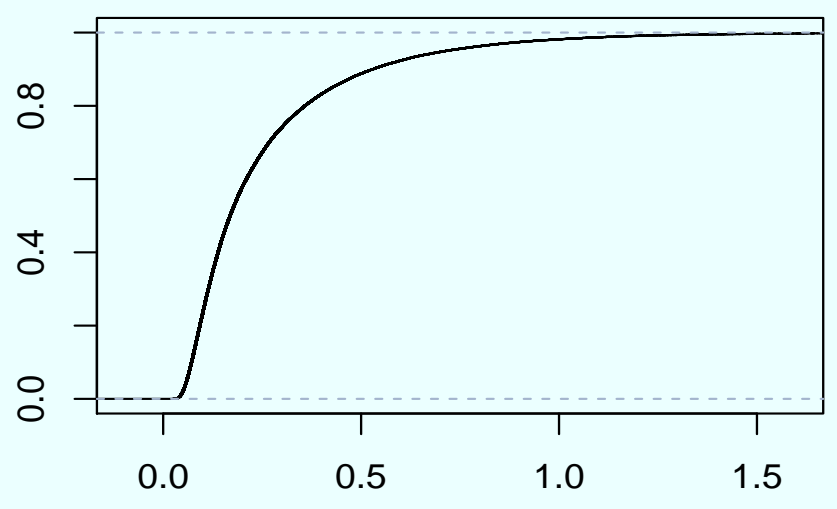

Fig.6: Empirical distribution function of $G_{2}(x)$

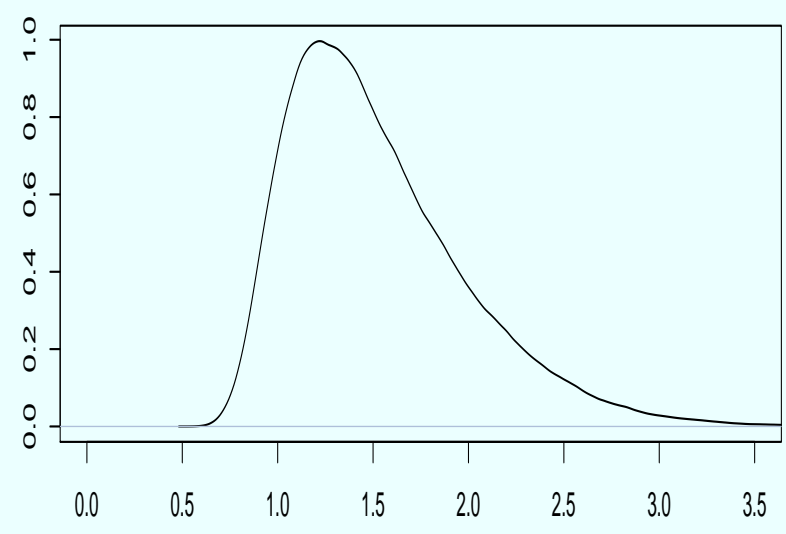

Fig.5: Kernel density estimation of $F_{2}^{\prime}(x)$

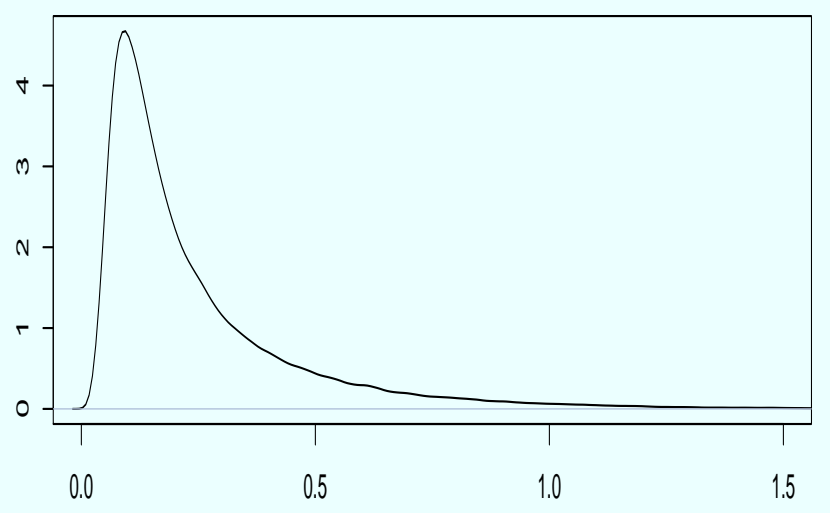

Fig.7: Kernel density estimation of $G_{2}^{\prime}(x)$ 


\section{References}

[1] R. J. Adler, The Geometry of random Fields, Wiley, Chichester, 1980.

[2] R. J. Adler, Continuity, extrema, and related topics for general Gaussian processes, IMS Lecture Notes - Monograph Series, Vol. 12, 1990.

[3] P. J. Bickel and M .J. Wichura, Convergence criteria for multiparameter stochastic processes ad some applications, Ann. Math. Statist. 42, 1656 - 1670, 1971.

[4] P. Billingsley, Convergence of Probability Measures, 2nd ed., Wiley, Chichester, 1999.

[5] E. Bolthausen, On the central limit theorem for stationary mixing random fields, Ann. Probab. 10, 1047 - 1050, 1982.

[6] A. G. Chedwynd and P. J. Diggle, On estimating the reduced second moment measure of a stationary spatial point process, Austral.\& New Zealand J. Statist. 40, 11 - 15, 1998.

[7] L. H. Chen and Q.-M. Shao, Normal approximation under local dependence, Ann. Probab. 32 (3A), 1985 - 2028, 2004.

[8] S. N. Chiu, D. Stoyan, W. S. Kendall and J. Mecke, Stochastic Geometry and Its Applications, 3nd ed., Wiley \& Sons, Chichester, 2013.

[9] D. J. Daley and D. Vere-Jones, An Introduction to the Theory of Point Processes, Vol. I: Elementary Theory and Methods, 2nd ed., Springer, New York, 2003.

[10] D. J. Daley and D. Vere-Jones, An Introduction to the Theory of Point Processes, Vol. II: General Theory and Structure, 2nd ed., Springer, New York, 2008.

[11] P. Deheuvels and G. Peccati and M. Yor, On quadratic functionals of the Brownian sheet and related processes, Stoch. Proc. Appl. 116, 493-538, 2006.

[12] B. J. Diggle, An Statistical Analysis of Spatial Point Patterns, 2nd ed., Arnold, London, 2003.

[13] P. Grabarnik and S. N. Chiu, Goodness-of-fit test for complete spatial randomness against mixtures of regular and clustered spatial point processesNormal approximation under local dependence, Biometrika 89, 411 - 421, 2002.

[14] L. Heinrich, Asymptotic normality of a point field characteristic in $R^{d}$, statistics 17, 453 - 460, 1986.

[15] L. Heinrich, Asymptotic Gaussianity of some estimators for reduced factorial moment 
measures and product densities of stationary Poisson cluster processes, statistics 19, 87 - 106, 1988.

[16] L. Heinrich, Goodness-of-fit tests for the second moment function of a stationary multidimensional Poisson process, statistics 22, 245 - 268, 1991.

[17] L. Heinrich and Z. Pawlas, Weak and strong convergence of empirical distribution functions from germ-grain processes, Statistics 42, 49 - 65, 2008.

[18] L. Heinrich, Asymptotic methods in statistics of random point processes, In E. Spodarev (Ed.), Stochastic Geometry, Spatial Statistics and Random Fields, Lecture Notes in Mathematics, Vol. 2068, Springer, New York, 2013, pp. 115 - 150.

[19] L. Heinrich, Asymptotic goodness-of-fit tests for stationary point processes based on scaled empirical K-functions, To be submitted, 2014.

[20] L. Heinrich, S. Lück and V. Schmidt, Non-parametric asymptotic statistics for the Palm mark distribution of $\beta$-mixing marked point processes, arXiv:12055044v1 [math.ST], 22 May 2012, 33 pages.

[21] L. Heinrich, S. Lück and V. Schmidt, Asymptotic goodness-of-fit tests for the Palm mark distribution of stationary point processes with correlated marks", Bernoulli, DOI: 10.3150/13-BEJ523, To appear 2015.

[22] L. P. Ho and S. N. Chiu, Testing the complete spatial randomness by Diggle's test without an arbitrary upper limit limit theorems for point processes, J. Statist. Comput. Simul. 76, 585 - 591, 2006.

[23] J. Illian, A. Penttinen, D. Stoyan and H. Stoyan, Statistical Analysis and Modelling of Spatial Point Processes, Wiley, Chichester, 2008.

[24] S. R. Jammalamadaka and S. Janson, Limit theorems for a triangular scheme of Ustatistics with applications to interpoint distances, Ann. Probab. 14, 1347 - 1358, 1986.

[25] ,E. Jolivet, Central limit theorem and convergence of empirical processes for stationary point prozesses, In P. Bartfai and J. Tomko (Eds.), Point Processes and Queuing Problems, Colloquia Mathematica Societatis János Bolyai, No. 24, Keszthely, Hungary, 1978, North-Holland, Amsterdam, 1980, pp. 117 - 161.

[26] A. F. Karr, Point Processes and Their Statistical Inference, Dekker, New York, 1986.

[27] A. F. Karr, Estimation of Palm measures of stationary point processes, Probab. Th. Rel. Field 74, 55 - 69, 1987. 
[28] K. Kiêu and M. Mora, Estimating the reduced moments of a random measure, Adv. Appl. Prob. 31, 48 - 62, 1999.

[29] E. N. Krivyakov, G. V. Martynov and Yu. N. Tyurin, On the distribution of the $\omega^{2}-$ statistics in the multi-dimensional case, Theory Probab. Appl. 22, 406 - 410, 1978.

[30] G. Last, M. D. Penrose, M. Schulte and C. Thäle, Moments and central limit theorems for some multivariate Poisson functionals, Adv. Appl. Prob. 46, 348 - 364, 2014.

[31] A. M. Liebetrau, The weak convergence of a class of estimators of the variance function of a two-dimensional Poisson process, J. Appl. Prob. 15, 433 - 439, 1978.

[32] G. V. Martynov, Computations of the distribution functions of quadratic forms of normal random variables, Theory Probab. Appl. 20, 797 - 809, 1975.

[33] G. V. Martynov, Omega-Square Criteria, Nauka, Moscow, 1978. (in Russian)

[34] G. Neuhaus, On weak convergence of stochastic processes with multidimensional time parameter, Ann. Math. Statist. 42, 1285 - 1295, 1971.

[35] J. Ohser and D. Stoyan, On the second-order and orientation analysis of planar stationary point processes, Biom. J. 23, 523 - 533, 1981.

[36] M. Reitzner and M. Schulte, Central limit theorems for U-statistics of Poisson point processes, Ann. Probab. 41, 3879 - 3909, 2013.

[37] D. B. Ripley, The second-order analysis of sttaionary point processes, J. Appl. Prob. 13, $255-266,1976$.

[38] R. J. Serfling, Approximation Theorems of Mathematical Statistics, Wiley, New York, 1980.

[39] M. Stein, A new class of estimators for the reduced second moment measure of point processes, Biometrika 78, 281 - 286, 1991.

[40] M. Stein, Asymptotically optimal estimation for the reduced second moment measure of point processes, Biometrika 80, 443 - 449, 1993.

[41] D. Stoyan and H. Stoyan, Improving ratio estimators of second-order point process characteristics, Scand. J. Statist. 27, 614 - 656, 2000.

[42] D. L. Zimmerman, A bivariate Cramér-von Mises type of test for spatial randomness, Appl. Statist. 42, 43 - 54, 1993. 


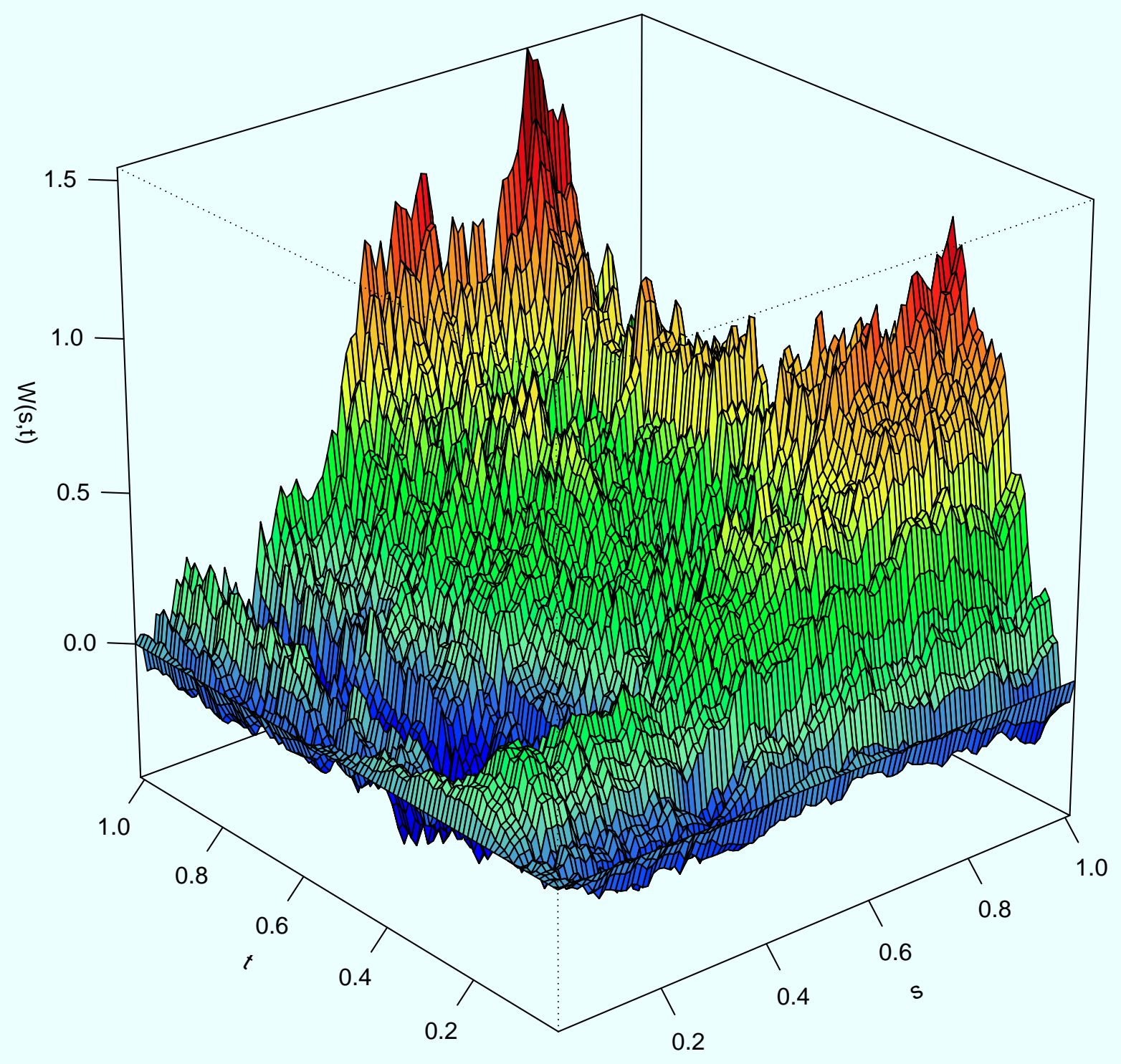

Fig.8: Wiener sheet over the unit square simulated on an equidistant $100 \times 100$ grid 